\title{
Coherent phonons in condensed media
}

\author{
Thomas Dekorsy, Gyu Cheon Cho and Heinrich Kurz \\ Institut für Halbleitertechnik, Rheinisch-Westfälische Technische Hochschule Aachen, \\ Sommerfeldstr. 24, D-52056 Aachen, Germany
}

\begin{abstract}
The impulsive excitation and phase-sensitive detection of coherent phonons and phonon-polaritons provide a detailed insight into the dynamical properties of matter. The experiments are based on optical pump-probe techniques with femtosecond time resolution. These techniques enable the detection of amplitude and phase of the coherent lattice motion simultaneously. Frequencies in the $\mathrm{THz}$ range and dephasing times in the picosecond range can be obtained with high accuracy. Especially in semiconductors and semiconductor heterostructures, where a coherent phonon mode and free carriers are excited simultaneously, important information about carrier-phonon interaction far away from equilibrium is obtained. This paper presents an overview of recent achievements in this vivid field of condensed matter physics.
\end{abstract}




\section{Contents}

Coherent phonons in condensed media

Thomas Dekorsy, Gyu Cheon Cho and Heinrich Kurz . . . . . . . . . 1 


\section{Introduction}

The time-resolved detection of transient phenomena in the picosecond and subpicosecond range has been enabled by the development of ultrafast laser sources. Ultrafast laser systems allow us to perform pump-probe experiments in several configurations concerning the photon energy, the light intensity, polarization selectivity, etc., thus a huge variety of impulsively stimulated processes can be studied in condensed media. Ultrafast time-resolved optical techniques contributed to disentangle the time constants of the most important interactions in semiconductors and semiconductor heterostructures. A thorough review of the whole field has been given recently by Shah [1].

First time resolved observations of non-equilibrium phonon dynamics were performed by means of time-resolved Raman scattering techniques [2, 3, 4]. By time-resolving the intensity of the anti-Stokes line, these experiments yield information on the decay of coherently excited phonon populations. The requirements for time-resolving a Stokes or anti-Stokes Raman peak is that the energetic pulse width must be smaller than the phonon energy, i.e. the pulse duration must be larger than a phonon period. Otherwise the observed phonon line would lie within the spectrum of the laser, aggravating the detection of the Raman shifted intensity. Therefore these techniques do not allow the resolution of the coherent phonon amplitude phase sensitively. Extensive investigation of the phonon dynamics in InP and GaAs have been performed by this technique by Vallée at al. [5, 6, 7]. For a review on coherent anti-Stokes Raman scattering techniques and results we refer to Bron [8].

Laser pulses with durations sufficiently shorter than the inverse of a fundamental lattice vibration frequency allow us to excite this lattice mode coherently, i.e. with a large number of phonons in one mode with constant phase relation [9]. This coherently excited mode is associated with a macroscopic lattice distortion. This chapter deals with the mechanisms responsible for the generation of coherent phonons and the different experimental possibilities for their detection. The focus lies on the excitation of opaque semiconductors. Analogies and differences to the well established techniques of Raman spectroscopy will be outlined. The experiments performed on coherent phonons within the last years have enlarged our knowledge on non-equilibrium states of phonons complementary to the knowledge gained by the mature and highly sophisticated field of inelastic light scattering in solids, for which the contributions to this series are outstanding references.

One important difference between Raman spectroscopy and time-resolved coherent phonon spectroscopy is the fact that in the latter the excitation and detection processes can be separated and that they may have different physical origins. In the detection process of coherent phonons, infrared-active and Raman-active act differently. In the case of polar lattices, a macroscopic polarization is generated by IR-active phonons at the phonon frequency, which leads to the emission of $\mathrm{THz}$ radiation $[10,11,12]$. Such a macroscopic polarization at phonon frequencies can be detected time resolved via nonlinear 
optical processes such as the first-order electro-optic effect (related to the second order nonlinear susceptibility $\chi^{(2)}$ ) [13] and the Franz-Keldysh effect (related to $\chi^{(3)}$ ) [14] in bulk semiconductors. Detection processes based on the quantum confinement of electronic states become relevant in twodimensional semiconductor heterostructures and quantum dots. For nonpolar lattice modes, i.e. only Raman-active modes, the detection process is closely related to the Raman susceptibility. In this case, the macroscopic lattice distortion leads to a change in reflectivity or transmission based on the Raman tensor $(\partial \chi / \partial Q) Q$, where $Q$ is the atomic displacement coordinate. While this detection is conceptually very close to inelastic light scattering experiments, there exist some fundamental differences: Let us assume a time interval of at least one phonon period for the inelastic interaction of a photon and a phonon in order to impose a sideband at the phonon frequency $\pm \omega_{\text {phonon }}$ on the light at frequency $\omega$. A sufficiently short laser pulse can be regarded as a delta-function in time compared to the oscillation period of the phonon, thus it represents a stroboscopic illumination of the crystal in a certain coherent displacement state $Q$. The intensity changes of the transmitted or reflected probe pulse do not necessarily obey the restrictions of wavevector and energy conservation for an inelastic scattering process.

This chapter is organized as follows: The generation and detection processes for coherent phonons are outlined in Sec. 2 and Sec. 3, respectively. The coherent phonon generation, detection, and manipulation by fs laser pulses is discussed in detail in Sec. 4 for the case of GaAs, since GaAs plays the role of a key material for the understanding of a non-Raman excitation process for coherent LO phonons in opaque semiconductors. Section 5 discusses the observation of coherent phonons and coupled phonon-carrier excitations in low-dimensional semiconductor heterostructures. Experiments performed on Te single crystals reveal the possibility to detect coherent phonons via their Raman activity or via their IR activity (Sec. 6). Thus it is shown that time-resolved optical pump-probe techniques and THz emission spectroscopy represent the counterpart to CW Raman and CW IR spectroscopy, respectively. High excitation experiments in Te allow the observation of an optically induced phase transition via monitoring the coherent phonon frequency. Results on phonon-polaritons in ferroelectric crystals and high-temperature superconductors are briefly discussed in Sec. 7. Finally, recent achievements in the field of coherent phonon spectroscopy are summarized in Sec. 8.

\section{Coherent phonon generation}

In this section we present a phenomenological description for the excitation of coherent phonons. The equation of motion of coherent phonons can be expressed as a driven harmonic oscillator for the coherent phonon displacement amplitude $Q[2,15]$ 


$$
\mu^{*}\left(\frac{\partial^{2} Q(t)}{\partial t^{2}}+2 \gamma_{\text {phonon }} \frac{\partial Q(t)}{\partial t}+\omega_{\text {phonon }}^{2} Q(t)\right)=F^{Q}(t),
$$

where $\mu^{*}$ a the reduced lattice mass, $\gamma_{\text {phonon }}$ a phenomenological damping constant, and $F^{Q}(t)$ the appropriate driving force. Equation (1) can be derived from a quantum mechanical model for the coherent phonon amplitude [9]. The damping constant $\gamma_{p h o n o n}$ is related to the dephasing time $T_{2}$ of the coherent mode via $\gamma_{p h o n o n}=1 / T_{2}$. The dephasing time $2 / T_{2}=1 / T_{1}+1 / T_{p}$ is a combination of phase-destroying contributions $\sim T_{p}$ and truly population decreasing contributions $\sim T_{1}[16]$. The latter are dominated by anharmonic decay processes, e.g. the decay of LO phonons into acoustic phonons. Other population decreasing processes are based on electron-phonon interaction, e.g. the absorption of a phonon by an electron.

The driving force $F^{Q}(t)$ is exerted by an ultrashort laser pulse. Different types of driving forces have been classified into Raman type and non-Raman type excitation [17]. The Raman driving force is given in lowest order by the interaction of the electromagnetic field $E$ and the Raman polarizability $(\partial \alpha) /(\partial Q)$ of the medium,

$$
F^{Q}(t)=\frac{1}{2}\left(\frac{\partial \alpha}{\partial Q}\right)_{k l} E_{k} E_{l}
$$

Another useful distinction can be made into impulsive and resonant type of excitation. An impulsive excitation of the phonon mode is explained by a model denoted as displacive excitation of coherent phonons (DECP) [18, 19, 20], which is based on an interband excitation from bonding to antibonding orbitals. This process leads to an impulsive change of the equilibrium position of the atoms and thereby to a coherent excitation of lattice vibrations maintaining the crystal symmetry, i.e. $A_{1}$ modes for a large variety of materials. Displacively excited coherent phonons are characterized by a $\cos \left(\omega_{\text {phonon }} t\right)$ dependence of the coherent amplitude [20]. DECP has been treated in a more rigorous quantum-mechanical description by Kuznetsov and Stanton [9]. DECP is one of the most prominent examples for non-Raman excitation mechanisms of coherent phonons. Further non-Raman mechanisms will be discussed later. For a comprehensive distinction of Raman and non-Raman processes we also refer to the recent review by Merlin [17].

Impulsive resonant excitation can be accomplished by the combined action of two field components, $E_{k}$ and $E_{l}$, delivered by the broad spectrum of the ultrashort laser pulse. According to wavevector and energy conservation rules a lattice mode with frequency $\omega_{l}-\omega_{k}=\omega_{\text {phonon }}$ and wavevector $\mathbf{k}_{l}-$ $\mathbf{k}_{k}=\mathbf{k}_{\text {phonon }}$ is excited. This process is denoted as impulsive stimulated Raman scattering (ISRS) [21, 22, 23, 24, 25]. The phonon wavevector can be manipulated by using two excitation pulses with well defined angles of incidence. The initial phase of the coherent lattice displacement excited via ISRS should obey a $\sin \left(\omega_{\text {phonon }} t\right)$ dependence, however, resonant ISRS may also exhibit a $\cos \left(\omega_{\text {phonon }} t\right)$ dependence [25]. ISRS is one prominent Raman 
mechanism for the excitation of coherent phonons, which has been exploited most frequently for the generation of coherent phonons in the past.

For polar Raman-active LO phonons the driving force in Eqn. (1) can be described in the following way [15]:

$$
F_{j}^{Q}(t)=R_{j k l} E_{k} E_{l}-\frac{e^{*}}{\epsilon_{\infty} \epsilon_{0}} P_{j}^{N L},
$$

where $R_{i j k}$ is the Raman tensor and $P_{j}^{N L}$ is a nonlinear longitudinal polarization along direction $j$. The nonlinear polarization $P_{j}^{N L}$ can be divided into several possible contributions [26]

$$
\begin{aligned}
P_{j}^{N L}= & \chi_{j k l}^{(2)} E_{k} E_{l}+\chi_{j k l m}^{(3)} E_{k} E_{l} E_{m} \\
& +\int_{-\infty}^{t} d t^{\prime} J_{j}\left(t^{\prime}\right)+N e \int_{-\infty}^{t} d t^{\prime} \int_{-\infty}^{\infty} d x_{j}\left\langle\Psi\left(x_{j}, t^{\prime}\right)\left|x_{j}\right| \Psi\left(x_{j}, t^{\prime}\right)\right\rangle
\end{aligned}
$$

where the first two terms represent higher order nonlinear susceptibilities, e.g. field-induced Raman scattering. The third and fourth terms describe polarizations set-up by longitudinal intraband currents. The third term represents a longitudinal polarization generated by non-equilibrium currents with risetimes faster than the phonon period. They can impulsively excite coherent LO phonons in a current surge model for the screening of surface fields of III$\mathrm{V}$ semiconductors $[13,27,28,11]$ (see Sec. 4.1 ) or via the ultrafast build-up of electric Dember fields [29, 10, 30] (see Sec. 6.2). It should be noted that these ultrafast currents do not require coherence in the electronic system. The last term of Eqn. (4) describes the case of coherent electronic wavefunctions, which may set-up a macroscopic intraband polarization. If the level splitting of coherently superposed electronic excitations is close to the LO phonon resonance, i.e. the frequency of the intraband polarization is close to the phonon frequency, a direct interaction of the electronic polarization with the lattice polarization is expected [31].

For polar phonons the coupling of the lattice polarization given by a displacement $Q$ and an electronic polarization $P$ is given by a set of coupled equations [11]:

$$
\begin{gathered}
\frac{\partial^{2}}{\partial t^{2}} P_{j}+\gamma_{e l} \frac{\partial}{\partial t} P_{j}+\omega_{e l}^{2} P_{j}=\frac{e^{2} N}{\epsilon_{\infty} \mu^{*}}\left(E_{j}^{e x t}-4 \pi \gamma_{12} Q_{j}\right) \\
\frac{\partial^{2}}{\partial t^{2}} Q_{j}+\gamma_{p h o n o n} \frac{\partial}{\partial t} Q_{j}+\omega_{p h o n o n}^{2} Q=\frac{\gamma_{12}}{\epsilon_{\infty}}\left(E_{j}^{e x t}-4 \pi P_{j}\right) .
\end{gathered}
$$

$E_{j}^{e x t}$ is a macroscopic electric field which has to be determined self-consistently via the Poisson equation and describes, e.g., the surface field dynamics. $\gamma_{12}$ is a coupling constant given by $\gamma_{12}=\omega_{T O} \sqrt{\left(\epsilon_{0}-\epsilon_{\infty}\right) /(4 \pi)}$. The generalized phenomenological damping constant $\gamma_{e l}$ describes the damping of the electronic polarization, e.g. the momentum scattering time of a dense carrier 
plasma or the dephasing time of the intraband polarization of coherent electronic wavepackets. The frequency $\omega_{e l}$ is either the plasma frequency or the frequency of a coherent wavepacket oscillation. The driving force in Eqn. (5) and (6) is set-up by a combined action of the electric field dynamics and the polarization, which has been expressed in the different possible contributions in Eqns. (3) and (4).

Equations (5) and (6) describe coupled electron-phonon dynamics in a macroscopic sense. For the case of homogeneous densities and negligible damping, Eqns. (5) and (6) reproduce the well known dispersion branches of coupled plasmon-phonon modes for a one-component plasma [32]

$$
\omega_{P P M}^{ \pm}=\frac{1}{2}\left(\omega_{L O}^{2}+\omega_{P}^{2} \pm \sqrt{\left(\omega_{P}^{2}+\omega_{L O}^{2}\right)^{2}-4 \omega_{P}^{2} \omega_{T O}^{2}}\right)^{(1 / 2)}
$$

with the plasma frequency $\omega_{P}^{2}=\left(e_{0}^{2} N\right) /\left(\epsilon_{0} \epsilon_{\infty} m^{*}\right)$, with $N$ being the electron density and $m^{*}$ the effective mass. The associated plasmon-phonon coupled modes were first observed in Raman experiments in doped GaAs [33, 34]. In order to temporally resolve coherent plasmon-phonon dynamics we have to bear in mind that an optically excited plasma may be strongly inhomogeneous in real space and k-space. For minimizing the lateral real-space inhomogeneity due to the excitation with a Gaussian beam profile it is incumbent to use excitation spots larger than the probe spot size. The spatial inhomogeneity perpendicular to the excited surface in absorbing materials has also to be considered.

The wavevector of the excited phonon modes strongly depends on the excitation process. For experiments in transparent media when the coherent phonon excitation is based on first-order Raman processes, the wavevector is given by the wavelength $\lambda$ of the pump laser, i.e. $k=2 \pi n / \lambda$, where $n$ is the refractive index. In two-beam excitation configurations based on $\chi^{(2)}$ processes the coherent phonon wavevector can be selected via the angle between the two laser pulses (see Sec. 7.2). For opaque semiconductors where the excitation is mainly based on non-Raman processes, e.g. DECP or the field screening mechanism, the excited phonon wavevector is mainly determined by the imaginary part of the refractive index $\kappa$, i.e. $k=2 \pi \kappa / \lambda$. Since the dispersion of optical phonons is weak for small wavevectors, their $k$ cannot be determined from the observed frequency with high accuracy. This is not the case for acoustic zone-folded phonons in semiconductor superlattices (see Sec. 5.3) and acoustic modes in bulk materials excited at the edge of the Brillouin-zone [35], where the frequencies observed allow one to disentangle details of the excitation mechanism.

\section{Detection of coherent phonons}

There exist different possibilities to detect coherent phonons in the time domain: Two main classes of detection methods can be distinguished. One 
class is based on the detection of changes in the optical properties of the investigated material at the frequency of the laser light. The other class is based on the time-resolved detection of far-infrared light in the $\mathrm{THz}$ frequency range generated by coherent IR-active phonons. These two classes reflect the two historic continuous-wave methods for investigating phonon resonances, i.e. Raman spectroscopy and IR spectroscopy, respectively.

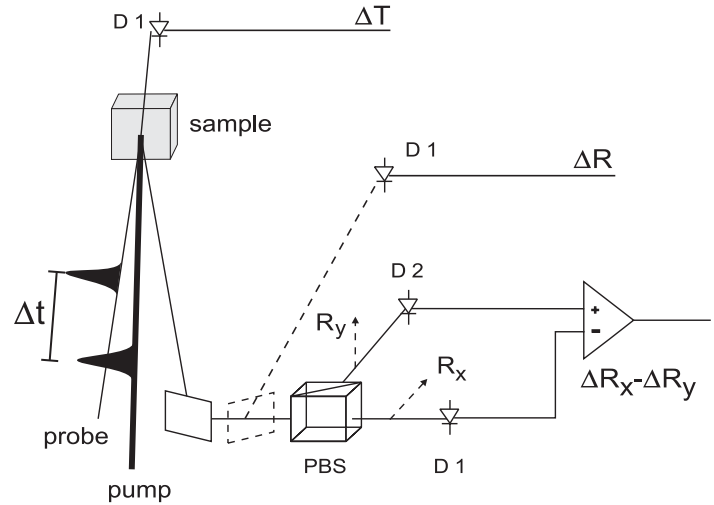

Fig. 1. Set-up for the time-resolved all-optical detection of coherent phonons in transient reflectivity $(\Delta R(\Delta t))$, transmission $(\Delta T(\Delta t))$, or polarization-analyzed reflectivity changes $\left(\Delta R_{x}(\Delta t)-\Delta R_{y}(\Delta t)\right) . D_{1}$ and $D_{2}$ denote photo-detectors and PBS a polarizing beam splitter.

The first class can be divided into the time-resolved detection of reflected, transmitted or diffracted light. The mechanisms giving rise to a modulation of the optical properties at the phonon frequencies can themselves be manifold. Figures 1 and 2 depict schematically the experimental setup and the principle for detecting coherent phonons in the time domain, respectively.

The modulation of the reflectivity $\Delta R$ can be explained on the base of the first-order Raman tensor $(\partial \chi) /(\partial Q)$, i.e.

$$
\Delta R=\frac{\partial R}{\partial n} \Delta n \sim \frac{\partial R}{\partial \chi} \frac{\partial \chi}{\partial Q} Q
$$

Since $(\partial \chi) /(\partial Q)$ is a tensor, different modes can be probed selectively by means of polarization analysis of the probe pulse [10, 36, 37]. For fully symmetric phonons the Raman tensor contains diagonal contributions of equal magnitude only. In this case the reflectivity changes will be isotropic irrespective of the polarization of the probe pulse relative to the crystal orientation. For phonon modes with non-diagonal terms in the Raman tensor, $(\partial \chi)(\partial Q)_{j k} \neq 0$ for $j \neq k$, the reflectivity changes will depend on the crystals orientation and the light polarization. Further detection schemes are based 


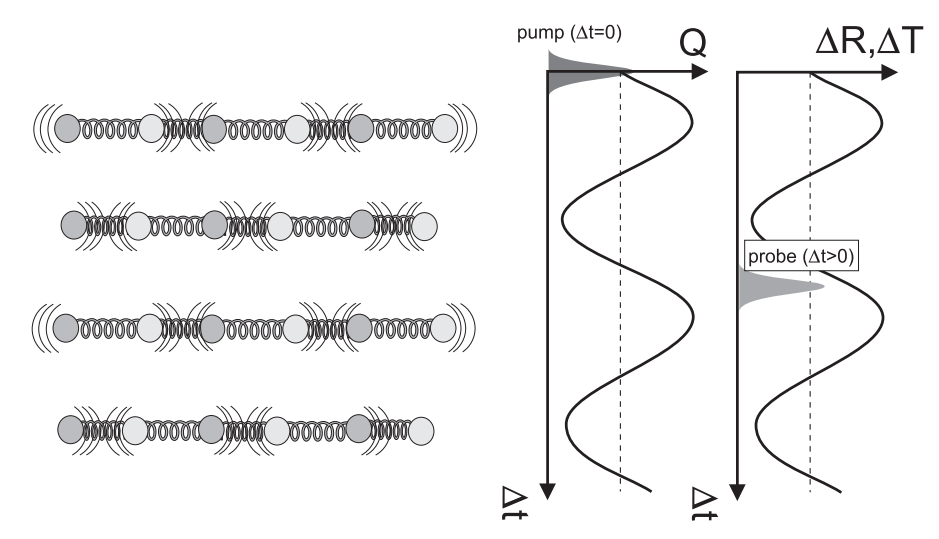

Fig. 2. Schematic sketch of the principle of time-resolved coherent phonon detection. The pump pulse induces a coherent atomic displacement $Q(\Delta t)$, which is stroboscopically sampled in the intensity changes of the reflected or transmitted probe pulse.

on Raman interaction with transmitted probe pulses, which results in a periodic shift of the spectral components of the probe pulse with the frequency of the lattice vibrations $[38,17]$. In crystals exhibiting a linear electro-optic effect (Pockels effect), coherent phonons may be detected sensitively via the associated longitudinal field [13]. In terms of Raman tensors, the so-called electro-optic contribution to the Raman tensor $(\partial \chi / \partial F)(\partial F / \partial Q) Q$ has to be added to Eqn. (8). The electric field induced phonon detection may be resonantly enhanced via third-order nonlinearities associated with the FranzKeldysh effect close to interband resonances [39, 14].

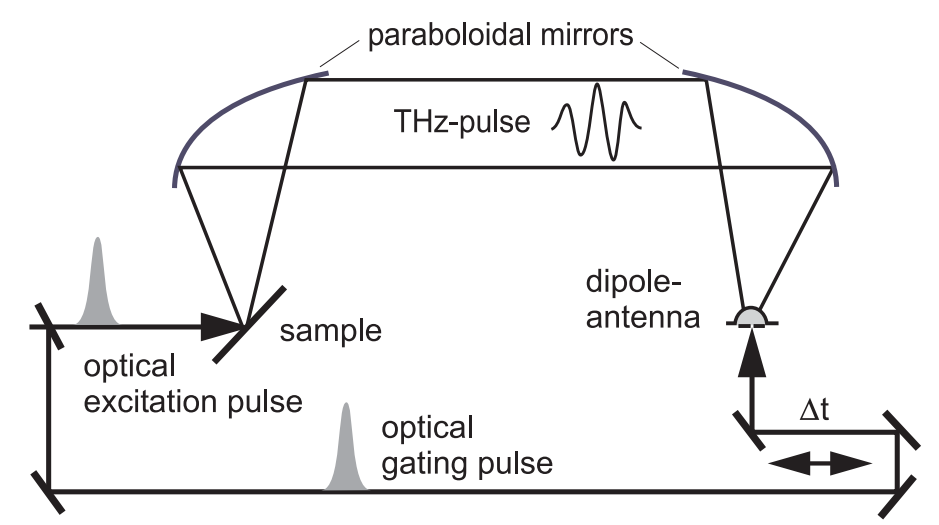

Fig. 3. Experimental set-up for the time-resolved detection of $\mathrm{THz}$ electromagnetic waves. 


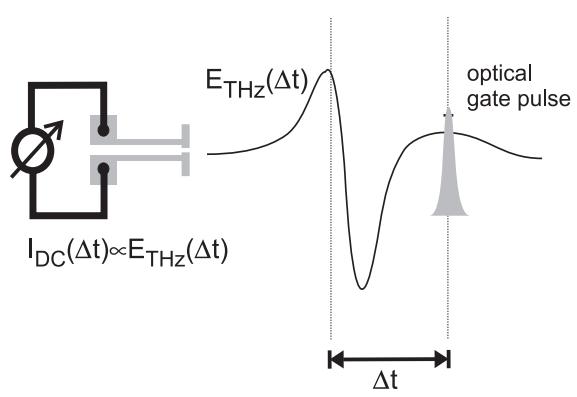

Fig. 4. Principle of the detection of $\mathrm{THz}$ electromagnetic pulses by using a gateable dipole antenna.

Another important possibility for detecting coherent IR-active phonons, phonon-polaritons or plasmon-phonon modes is via the emission of electromagnetic radiation in the $\mathrm{THz}$ frequency range. The emission characteristics can be assumed to follow the emission of a dipole, which is set-up by the volume in which coherent phonons form a macroscopic polarization. The pointing vector of the radiation is given by [11]

$$
\mathbf{S}(t)=\frac{\omega_{\text {phonon }}^{4} \mathbf{P}^{2}(t) \sin ^{2} \theta}{16 \pi^{2} \epsilon_{0} r^{2} c^{3}} \mathbf{s},
$$

where $\theta$ is the angle between the direction of observation $\mathbf{s}$ and the polarization $\mathbf{P}$, and $r$ is the distance to the detector. Experimentally, the timeresolved detection of $\mathrm{THz}$ electromagnetic fields has become possible by the application of ultrafast photoconductive switches [40]. Figures 3 and 4 depict the experimental arrangement for time-resolving $\mathrm{THz}$ electromagnetic fields. The emitted $\mathrm{THz}$ radiation is collected by paraboloidal mirrors onto the dipole antenna, which is activated by the time-delayed gating pulse. The antenna of approximately $50 \mu \mathrm{m}$ dipole length is fabricated on a semiconductor exhibiting free carrier lifetimes in the subpicosecond range, e.g. ionimplanted silicon on sapphire or low-temperature-grown GaAs. The incident $\mathrm{THz}$ electric field drives a current in the outer circuit of the antenna during the time span of the gate pulse. The measured current is directly proportional to the incident field strength. Thus the $\mathrm{THz}$ electric field can be measured in amplitude and phase. The bandwidth of the antennas is roughly given by the free carrier lifetime in the photoconductive switch $(<1$ ps depending on the material used). Useful frequency bandwidths are typically in the range of $100 \mathrm{GHz}$ to $3 \mathrm{THz}$. This limit excludes many interesting phonon modes in semiconductors to be studied. However, recent developments in the detection of $\mathrm{THz}$ radiation via electro-optic crystals are highly promising for achieving a detection bandwidth up to $30 \mathrm{THz}[41,42]$. 


\section{Coherent LO phonons in GaAs}

\subsection{Coherent phonon generation and detection in GaAs}

The coherent phonon generation in opaque semiconductors was first observed by Cho et al. in the time-resolved reflectivity changes in GaAs [13] and by Cheng at al. in $\mathrm{Bi}$ and $\mathrm{Sb}$ [18]. Here we focus on the experiments in GaAs, a material which has been intensively studied by Raman spectroscopy [43, 44]. The results obtained by fs spectroscopy on GaAs clearly demonstrate some of the main points of time-resolved phonon spectroscopy, i.e.

- the impulsive excitation via non-Raman effects due to the excitation of a dense electron-hole plasma in combination with built-in electric fields, i.e. the dynamics described by Eqn. (5) and (6),

- the visibility of coherent phonons via $\partial \chi / \partial Q$ according to the electrooptic Raman tensor and higher order terms,

- the dispersion of the detection process in the vicinity of singularity points of the band structure, e.g. the resonance enhancement of the FranzKeldysh effect,

- the coupling between coherent LO phonons and non-equilibrium carrier distributions.

The laser system employed in the first subpicosecond time-resolved experiments on GaAs was a colliding pulse modelocked (CPM) dye laser which delivers light pulses at $2 \mathrm{eV}$ with pulse durations of $50 \mathrm{fs}$. For the detection of coherent phonons, which often give small contributions on a large electronic background signal, a special fast-scanning detection system has been developed $[45,13]$. This detection system is based on scanning the time-delay between pump and probe pulses with a retroreflector mounted onto an activated vibrator. This allows the scanning of the time-delay at frequencies around $100 \mathrm{~Hz}$. With a real-time data acquisition system based on a homebuilt VME-bus computer system the data are accumulated without using further lock-in filtering techniques. This technique enabled the detection of time-resolved signal changes down to some $10^{-7}$ even with the CPM dye laser as pulse source [46].

In order to explain the excitation mechanism for coherent phonons in GaAs, electric surface fields at bare surfaces of III-V compounds have to be taken into account. Charged surface states lead to a pinning of the Fermi level within the gap, in turn producing a bending of the band structure towards the surface. The associated electric fields are calculated within a Schottkybarrier model [47] and exhibit a square root dependence on the doping density of the crystal. An external manipulation of the surface field is possible via transparent Schottky contacts. Figure 5 sketches the underlying process for the generation of coherent LO phonons in GaAs surface space charge fields. The optical injection of carriers within the surface field region leads to an ultrafast current surge which rapidly screens the built-in electric field [28]. 


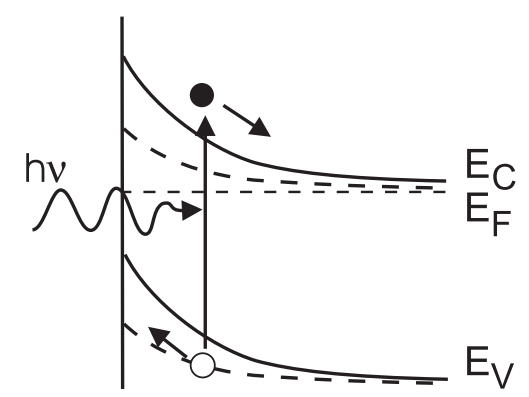

Fig. 5. Schematic sketch of the field screening process at a bare surface of an n-doped semiconductor with mid-gap Fermi-level pinning at the surface. The solid (dashed) lines represent the band bending before (after) the optical excitation.

Associated with this ultrafast polarization change is the emission of broadband $\mathrm{THz}$ radiation [40]. The ultrafast depolarization of the surface depletion field leads to the coherent excitation of LO phonons [13, 27]. The detection for wavelengths far from resonances is accomplished via the Pockels effect [48]. On (100) GaAs surfaces, a longitudinal field along the [100] direction leads to induced birefringence in the (100) plane. By subtracting two polarizations components of a linearly polarized probe pulse along the [011] and [011] direction, the reflectivity change is given by $[49,13]$

$$
\frac{\Delta R(t)}{R_{0}}=\frac{\Delta R_{[011]}(t)-\Delta R_{[01 \overline{1}]}(t)}{R_{0}}=\frac{4 r_{41} n_{0}^{3}}{n_{0}^{2}-1} \Delta E_{\text {surface }}(t) .
$$

where $r_{41}$ is the electro-optic coefficient, $n_{0}$ is the unperturbed refractive index, and $\Delta E_{\text {surface }}(t)$ is the time dependent macroscopic electric surface field along the [100] direction.

Figure 6 depicts the electro-optic reflectivity changes of an n-doped GaAs sample prepared with a transparent indium-tin oxide Schottky contact on top. The optical excitation density is $4 \times 10^{17} \mathrm{~cm}^{-3}$, the experiments were performed at room temperature. The transparent Schottky contact allows the change of the electric surface field by applying different voltages to the sample. The electro-optic measurements in Fig. 6 reveal the transient screening of the surface field, $\Delta E_{\text {surface }}(t)$,for different initial surface field strengths. The screening dynamics exhibit a fast component on the time scale of the exciting pulse, i.e. within one optical phonon period, and a slower component on a ps time scale. The associated electric field changes are in the order of $100 \mathrm{kV} / \mathrm{cm}$ [28]. The data are clearly modulated with oscillations with a frequency of $8.75 \mathrm{THz}$, matching the GaAs $\mathbf{k}=0 \mathrm{LO}$ phonon frequency at 300 $\mathrm{K}$. The dephasing time of the oscillations, $4.0 \pm 0.3 \mathrm{ps}$, is independent of the applied electric fields. This dephasing time is in agreement with the phonon lifetime derived from CARS experiments [5]. This agreement suggests that the observed decay of the coherent amplitude is determined by anharmonic 


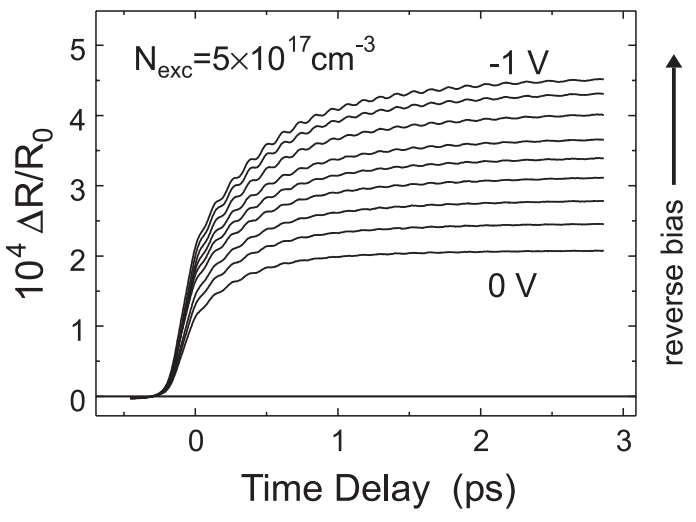

Fig. 6. Electro-optic reflectivity changes from (100) GaAs with a transparent Schottky contact on top. The data are obtained for different reverse-bias voltages at 300 K (from Ref. [28]).

decay and not by pure dephasing processes. Such an agreement between the dephasing time derived from coherent phonon experiments and the linewidth obtained in Raman scattering experiments has also recently been observed in a thorough study on the dephasing in single-crystal bismuth [50].

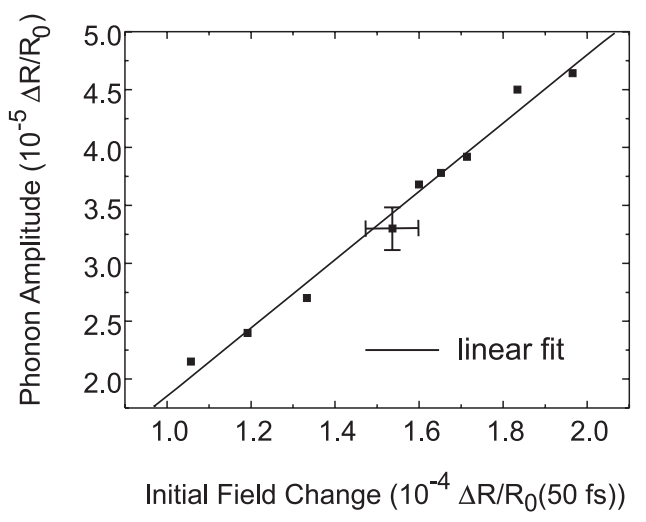

Fig. 7. Amplitude of coherent LO phonons (solid squares) versus the electro-optic reflectivity changes in Fig. 6 at a time delay of 50 fs (from Ref. [27]).

In order to verify that the field screening is the responsible driving force of the coherent LO phonons, we plot the phonon amplitude versus the reflectivity change at a time delay of $50 \mathrm{fs}$ (Fig. 7). This value is proportional to the driving force for coherent LO phonons according to the third term in 
Eqn. (4), since it represents the optically detected surface field changes, i.e. $\Delta R(50 \mathrm{fs}) \sim \Delta E_{\text {surface }}(50 \mathrm{fs})$. The data reveal a linear dependence of the phonon amplitude on $\Delta R(50 \mathrm{fs})$, thus confirming the proposed generation mechanism. The initial phase of the oscillations is $\sim \cos (\omega t)$. This behavior is expected for an oscillator starting at a displaced position, i.e. the atomic positions at negative time delays, driven to oscillate around a new equilibrium position given by the electric field at positive time delays.

The excitation process of coherent LO phonons observed in GaAs is isotropic in the sense that the coherent phonon amplitude is independent of the incident polarization of the pump pulse [38, 27]. This observation rules out a coherent phonon generation via a $\chi^{(2)}$ process (first right-hand term in Eqn. (4)). The last remaining Raman process which could in principle account for an isotropic generation process is a third-order nonlinear polarization with the third field being the surface field (second right-hand term in Eqn. (4)). This effect has been observed in CW Raman experiments close to electronic resonances and has been denoted as inverse Franz-Keldysh effect [51]. This effect has been shown to drop in scattering efficiency by orders of magnitude when the laser energy is detuned from interband resonances by a few tens of meV. This effect could not be ruled-out completely as generation mechanism at the time when only fs lasers with $2 \mathrm{eV}$ photon energy were available. Therefore this question will be addressed later in the context of resonant excitation with a tunable fs titanium-sapphire laser.

Intriguing situations in light scattering experiments arise when the photon energy is in the vicinity of interband resonances [52, 53, 39]. The invention of the Kerr-lens modelocked Ti:sapphire laser allows one now to perform coherent phonon experiments over a wide range of excitation wavelengths (from $700 \mathrm{~nm}$ to $1000 \mathrm{~nm}$ ). This range covers the bandgap of GaAs $(1.42 \mathrm{eV}$ at room temperature). Hence different contributions to the resonant and nonresonant excitation and detection of coherent phonons can be investigated.

Figure 8 shows the time resolved reflectivity change from a (100) GaAs surface for $2.0 \mathrm{eV}$ and $1.47 \mathrm{eV}$ laser energy obtained with two different probe polarizations along the principle axis of the electro-optic index ellipsoid, i.e. the [011] and [011] rystal direction. In contrast to the electro-optic reflectivity changes shown in Fig. 6, the probe pulse is not polarization analyzed. The observed reflectivity changes at $2 \mathrm{eV}$ have two contributions: (i) An electronic contribution due to the refractive index changes/absorption changes associated the free carriers introduced by the excitation of interband transitions. This contribution is isotropic in the sense that it does not depend on the probe pulse orientation. (ii) The electro-optic contribution which is of comparable magnitude as the isotropic reflectivity change. For a probe polarization along the [011] ([011]) crystal direction the electro-optic effect gives a positive (negative) contribution to the carrier-induced reflectivity changes. This sign reversal of the electro-optic contribution is also observed for the phonon-induced contribution, as can be seen in the $\pi$ phase shift between 

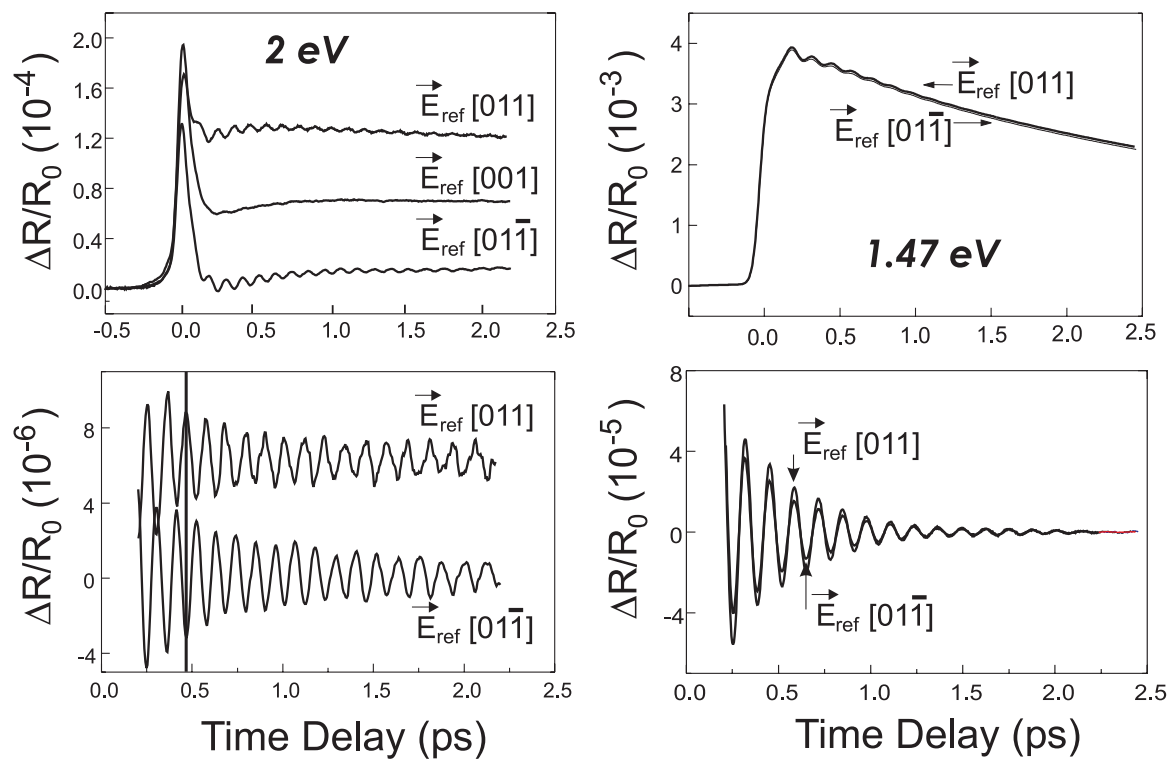

Fig. 8. Comparison of reflectivity changes of (100) GaAs at $2 \mathrm{eV}$ (left) and 1.47 $\mathrm{eV}$ (right) photon energy for different polarizations of the reflected probe beam $\mathbf{E}_{\text {ref }}$, i.e. along the [001], the [011], and the [011] direction. In the lower part the oscillatory contributions are compared for the [011] and the [011] polarization.

the oscillations. The same measurements performed close to the band edge exhibit a much larger total reflectivity change and a phonon induced contribution larger by more than an order of magnitude than the electro-optic contribution at $2 \mathrm{eV}$. In addition the phase of the oscillations does not change with the probe polarization. Only a tiny difference exists between the two curves with a magnitude comparable to the difference between the two equivalent $2 \mathrm{eV}$ curves. All these observations point toward the fact that the phonon detection at the fundamental bandgap is not based on the electro-optic effect but on an isotropic optical nonlinearity. This contribution arises from a third order nonlinearity associated with the Franz-Keldysh effect [14]. The term in expansion of the nonlinear third-order polarization responsible for this detection process is

$$
P(\omega)=\epsilon\left(\chi^{(3)} E_{\text {surface }}(0) E_{\text {phonon }}\left(\omega_{L O}\right) E(\omega)\right)
$$

where $E_{\text {surface }}(0)$ is the surface field, $E_{\text {phonon }}\left(\omega_{L O}\right)$ the field associated with the coherent LO phonons, and $E(\omega)$ the light field. The surface field $E_{\text {surface }}(0)$ is assumed to be nearly static with respect to the light frequency. However, the subpicosecond surface field dynamics also influences the coherent phonon amplitude detected via Eqn. (11). 


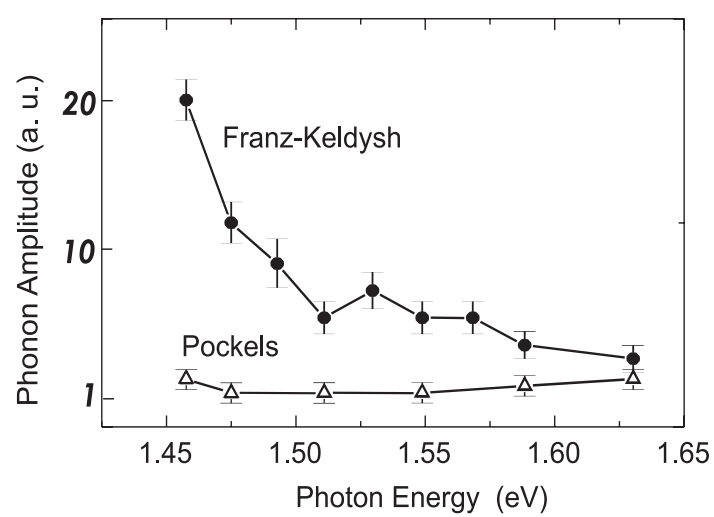

Fig. 9. Dispersion of the electro-optic (open squares) and Franz-Keldysh (closed dots) contribution to the coherent LO phonon amplitude (from Ref. [14]).

Figure 9 shows the dispersion of the electro-optic and of the Franz-Keldysh contribution derived from the detection of coherent LO phonons at different laser energies, i.e. the dispersion of $\chi^{(2)}$ and $\chi^{(3)}$ at the LO phonon frequency. The data reveal a nearly dispersion-free electro-optic effect, while the FranzKeldysh-like detection is strongly enhanced when the energy approaches the band edge. This data give clear evidence for higher order nonlinearities being relevant in the resonant detection of phonons. A dispersion of the generation process can be ruled out experimentally, since it would lead to a change in the phonon amplitude in the electro-optic detection scheme. Thus these measurements rule out the inverse Frank-Keldysh effect discussed above as a relevant excitation mechanism.

\subsection{Coupled plasmon-phonon modes}

The interaction of phonons and plasmons has been an intensively investigated subject in Raman experiments since the first observations in doped semiconductors by Mooradian and McWorther [34]. The origin of such coupled modes has already been discussed in Sec. 2. Time-resolved experiments allow the study of non-equilibrium effects on the coupled dynamics. We focus on experiments in GaAs, where coherent LO phonons are generated and detected as discussed in the previous section.

Figure 10 shows early data on coupled plasmon-phonon modes obtained in the electro-optic detection configuration with $2 \mathrm{eV}$ laser pulses delivered by a CPM laser [54]. The time domain data exhibit a pronounced beating which is identified as a superposition of a coherent LO phonon and a broad plasmon contribution centered around the TO phonon, i.e. the screened LO mode. No distinct modes other than LO or screened LO could be observed. This fact is a shortcoming of the output power delivered from the CPM laser. 


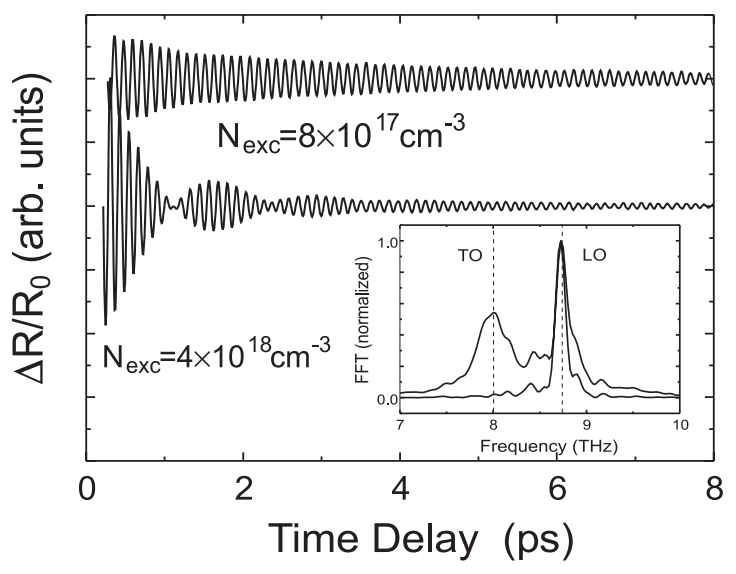

Fig. 10. Extracted phonon signature of electro-optic reflectivity changes obtained on (100) GaAs at $2 \mathrm{eV}$ excitation for the two different densities given in the figure. The inset shows the Fourier transforms of the time domain data.

In order to achieve high carrier densities in excess of $5 \times 10^{17} \mathrm{~cm}^{-3}$, necessary to drive the plasma frequency into resonance with the LO phonon, the pump spot has to be focused tightly to $<20 \mu \mathrm{m}$. At this focus size, the probe pulse averages over a lateral highly inhomogeneous carrier density, so that distinct plasmon modes cannot be resolved. Beside this spatial inhomogeneity, the optically generated plasma is also highly inhomogeneous in k-space. At $2 \mathrm{eV}$ excitation energy strong electronic intervalley transfer into $\mathrm{X}$ and $\mathrm{L}$ valleys of the conduction band within the first 100 fs has been reported [55]. This leads in turn to a multicomponent plasma not having a well defined frequency.

By using a Ti:sapphire laser, both lateral and k-space inhomogeneities can be suppressed. Figure 11 depicts coherent plasmon-phonon oscillations detected in the electro-optic reflectivity change at $1.5 \mathrm{eV}$. The crystal is (100) oriented n-doped GaAs with a doping density of $N_{D}=3 \times 10^{17} \mathrm{~cm}^{-3}$. The data are recorded using a 5 times larger pump than probe spot diameter. Thereby it is possible to scan the probe spot across the pump spot, thus detecting different optically excited densities. The maximum density in the center of the pump spot is $1 \times 10^{18} \mathrm{~cm}^{-3}$. At this point a rapidly dephasing mode with a frequency close to the screened LO phonon is observed. For detection spots further away from the center, the frequency of the fast dephasing component decreases according to the dispersion of the lower plasmon branch and the unscreened LO phonon dominates the reflectivity changes. These results demonstrate that the mode-beating observed at high densities using 2 $\mathrm{eV}$ excitation (Fig. 10) stems from different contributions within the laterally inhomogeneous plasma.

After having demonstrated the time resolved detection of coherent plasmonphonon modes we investigate the effect of a cold carrier plasma introduced 


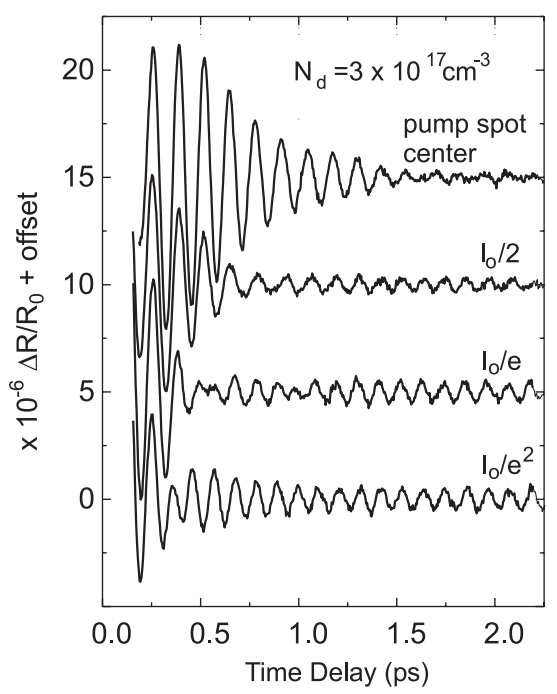

Fig. 11. Time-resolved phonon traces probed via the Pockels effect with a small probe spot scanned over a large Gaussian excitation spot (from Ref. [56]).
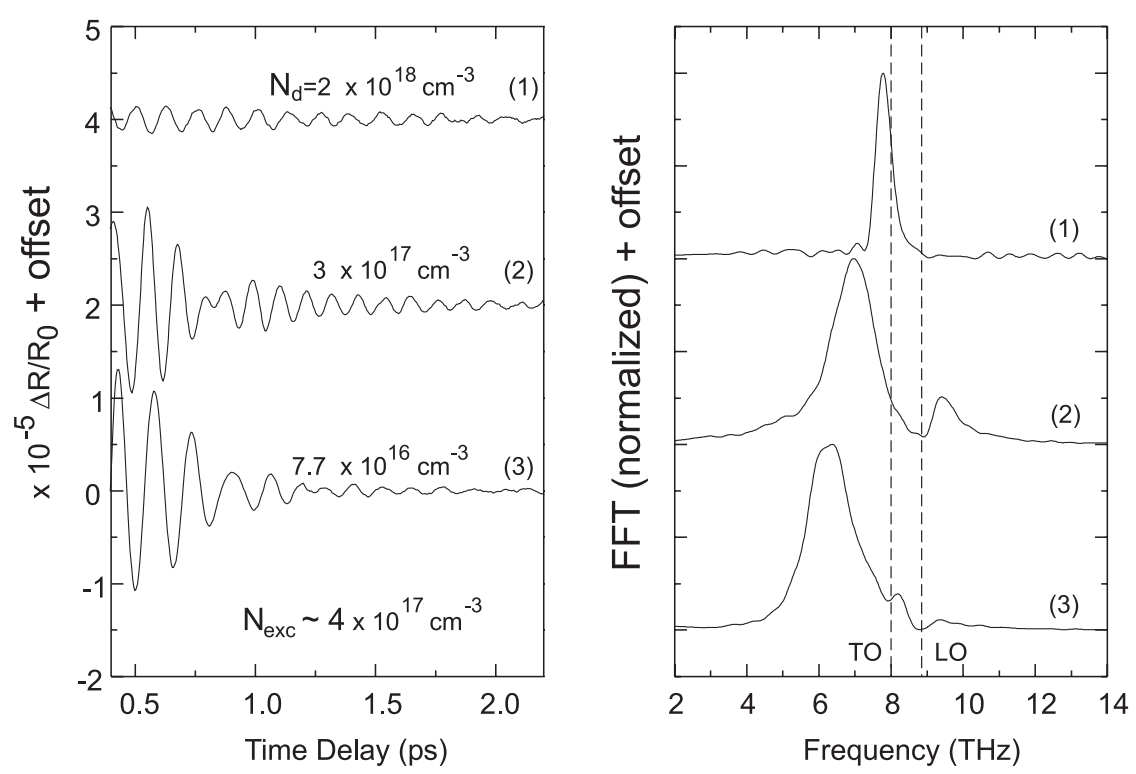

Fig. 12. Oscillatory part of isotropic reflectivity changes of differently doped GaAs samples at a constant excitation density (left) and Fourier transforms of the data (right, from Ref. [57]). 
via doping of the sample. This investigation is expected to shed light on the mechanism relevant for the dephasing of coherent plasmon oscillations [57]. Figure 12 shows coherent plasmon-phonon signatures from n-doped GaAs crystals with different doping densities at a constant optical excitation density. Figure 13 depicts the frequencies derived from the experimental data in comparison with the theoretical plasmon-phonon dispersion curve. Clearly, the data only follow the expected dependence if the sum over the optically excited density and the cold plasma resulting from the doping is taken into consideration. This observation gives strong evidence that the initially thermalized background plasma participates in the coherent plasmon oscillation. A closer analysis of the dephasing time of the plasmon oscillations reveals that it decreases if the relative contribution of the optically excited density increases, even when the total carrier density is kept at a constant level. This is a clear indication of the importance of electron-hole scattering for the plasmon dephasing [57].

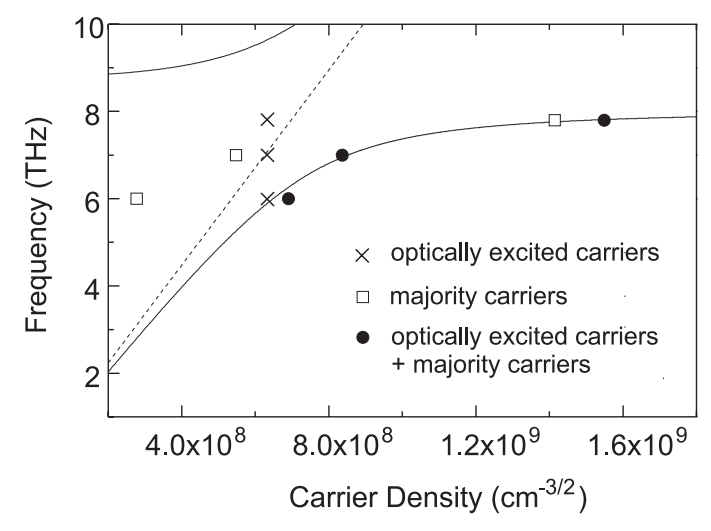

Fig. 13. Plasmon-phonon dispersion (solid line), plasmon frequency (dashed line) and the frequencies obtained from the time-resolved data as a function of the optically excited density (crosses), the doping densities of the sample (open squares) and the sum of both (closed circles) (from Ref. [57]).

Recently, $\mathrm{THz}$ radiation from coherent plasmon modes has been reported by Kersting et al. [58]. In time-resolved reflectivity experiments on GaAs at $77 \mathrm{~K}$, plasmons far away from the phonon resonance were detected by Sha et al. [59]. Furthermore, coherent plasmon-phonon modes as presented here were observed in time-resolved second harmonic generation (SHG) from bulk GaAs [60]. This detection is closely related to the electro-optic detection of plasmon-phonon modes presented here. Due to the possible selectivity of SHG towards the surface contribution, this technique opens an intriguing way to study the temporal dynamics of interface phonons [61, 62]. 


\subsection{Coherent control of LO phonons}

The phase information obtained from the time resolved detection of the coherent amplitude opens the way towards the coherent control of that amplitude. Multiple successive pulses have been applied for a selective enhancement of certain vibrational modes in molecular systems [63]. Especially the recent advances in the shaping of femtosecond pulses [64] open the way to drive the phonon dynamics in a well defined way. By this technique, lattice distortions can be achieved, which could not be accomplished with a single intense optical pulse due to the saturation of the optical transition.

The concept of coherent control over the phonon dynamics is readily illustrated by means of a two-pulse excitation experiment [65]. Figure 14 depicts the transient electro-optic reflectivity changes obtained on a (100) GaAs surface. The excitation of coherent LO phonons is achieved via the field screening mechanism. Two successive pump pulses impinge upon the sample. Their intensity and time delay is adjusted in such a way that the second pulse provides a driving force for a coherent amplitude equivalent to the amplitude persistent from the first pulse. In addition, the initial surface field is adjusted via a transparent Schottky contact, in order not to screen the surface field completely already with the first pulse. By carefully adjusting the pulse separation, the driving force is in-phase or out-of-phase with the primarily generated coherent mode. In this way, complete destruction or resonant enhancement of coherent LO phonons is observed. This method enables the generation of coherent LO phonons for a well defined time interval shorter than the intrinsic dephasing time of LO phonons. Similar experiments are performed in $\mathrm{Sb}$, where the $\mathrm{A}_{1}$ mode is manipulated in a similar way [37]. In $\mathrm{Bi}-\mathrm{Sb}$ mixed crystals, the $\mathrm{Bi}-\mathrm{Bi}, \mathrm{Bi}-\mathrm{Sb}$, and $\mathrm{Sb}-\mathrm{Sb}$ vibrations could be enhanced and canceled by applying femtosecond pulse trains [66]. Intriguing experiments have been reported on the coherent control over the dynamics of degenerate phonon modes, which allow even the generation of circularly polarized phonons [67]. Recently, the coherent control of acoustic phonons in superlattices was accomplished, where acoustic backfolded modes of different order were silenced out or enhanced by applying multiple successive pump pulses. By this method a high sensitivity detection of higher order modes can be achieved [68] (for the generation of acoustic phonons see Sec. 5.3).

\section{Coherent phonons in low-dimensional semiconductors}

\subsection{Coupled intersubband-plasmon phonon modes in quantum wells}

Carrier-phonon interaction is one of the most important processes leading to energy relaxation in semiconductor devices, e.g. in heterostructure lasers [69]. The relaxation channels are strongly modified compared to the bulk semiconductor due to the introduction of discrete energy levels. The relaxation rates 
(a)
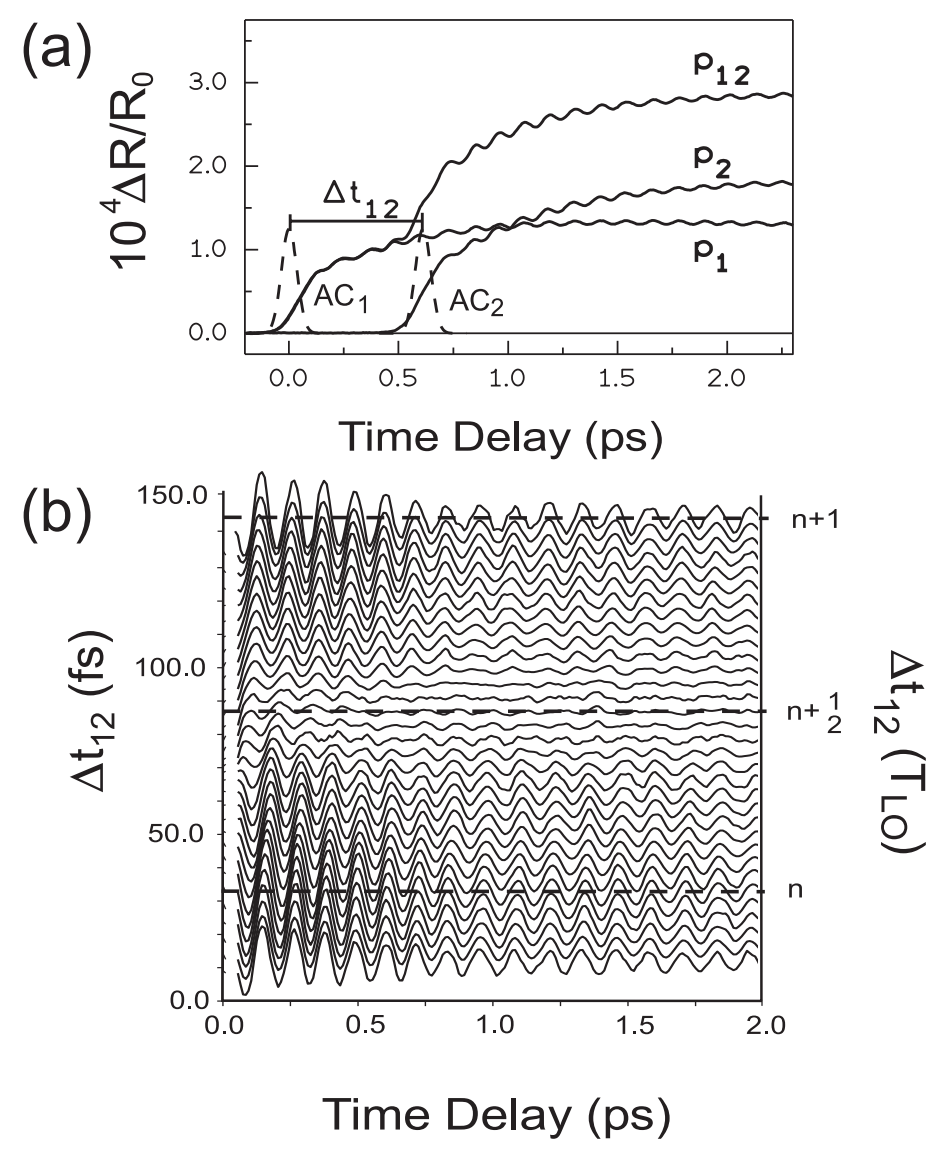

Fig. 14. (a) Electro-optic reflectivity changes of a (100) GaAs surface covered with an Indium-Tin-oxide Schottky contact on top. Two fs laser pulses at $2 \mathrm{eV}$ separated by the delay $\Delta t_{12}$ are used for coherent phonon generation. $p_{1}$ and $p_{2}$ denote the data obtained with single pulse excitation, $p_{12}$ the signal for double pulse excitation. (b) The extracted oscillatory contribution of the signal after the second pump pulse for different pulse separations $\Delta t_{12}$ in units of the LO phonon $\operatorname{period}(\mathrm{n}=4)$.

may strongly depend on whether the level spacing is smaller or larger than an LO phonon energy [70]. Especially at the high carrier densities present in semiconductor lasers during operation, new collective modes can be formed based on the coupling between intersubband transitions and phonons. Such modes have been denoted as intersubband-plasmon modes; they have been observed in Raman scattering experiments in two-dimensional electronic systems [71, 72, 73]. A review of the investigations of elementary excitations in these systems by inelastic light scattering has been given by Pinczuk and Abstreiter [74]. 
In the case of femtosecond excitation a non-equilibrium population of several subbands can be achieved. This non-equilibrium population alters the dielectric response of the system along the growth direction. If the associated change in the polarization is fast enough on the time-scale of the transition frequencies, the new resonances of the dielectric response are excited coherently and can be probed via the electro-optic detection scheme described in Sec. 4.1. We would like to note that effects based on field screening are strongly altered in $2 \mathrm{D}$ due to the confinement of the carriers along the growth direction of the heterostructures.

The dielectric response of the two dimensional system considering several subbands may be written as [75]:

$$
\epsilon(\omega)=\epsilon_{\infty}\left(\frac{\omega_{L O}^{2}-\omega^{2}}{\omega_{T O}^{2}-\omega^{2}}\right)+4 \pi \sum_{\alpha, i, j} \frac{\left|d_{i j}^{\alpha}\right|^{2}\left(n_{i}^{\alpha}-n_{j}^{\alpha}\right)}{\varepsilon_{i}^{\alpha}-\varepsilon_{j}^{\alpha}+\omega}
$$

where $\alpha$ denotes the heavy-hole and conduction band, $i$ and $j$ are the subband indices, $\epsilon_{i, j}$ are the subband energies, $d_{i j}^{\alpha}$ is the dipole matrix element between level $i$ and $j$, and $n_{i, j}^{\alpha}$ is the population of the subbands summed over perpendicular momenta. We note that the second part of Eqn. (12) gives a contribution to the dielectric function if $n_{i}^{\alpha} \neq n_{j}^{\alpha}$, i.e. two adjacent levels are populated differently. The electronic dielectric function has been calculated for different quantum well widths and under the assumption of a thermal population of the subbands [75].

Figure 15 depicts experimental results for $15 \mathrm{~nm}$ wide multiple quantum wells excited resonantly at the lowest interband transitions for three different excitation densities. The highest excitation density corresponds to $3 \times 10^{18} \mathrm{~cm}^{-3}$ in bulk GaAs. At this level the bulk coherent phonon spectra are fully dominated by the screened LO phonon at the TO frequency (see Sec. 4.2). In the quantum well, the screening is strongly suppressed, since the carriers cannot move freely along the (100) direction. With increasing excitation density in the quantum well a mode beneath but close to the TO frequency evolves, while the mode close to the LO phonon slightly shifts to higher energies. Both features can be qualitatively explained by the shift of the resonances introduced by a non-thermal population of the different subbands [75].

Further time-resolved experiments on plasmon-phonon coupling have been reported by Baumberg and Williams for a $\mathrm{GaAs} / \mathrm{Al}_{x} \mathrm{Ga}_{1-x} \mathrm{As} 2 \mathrm{D}$ electron gas [76]. An increase in the screened LO phonon at TO frequency was observed for increased optical excess energy above the GaAs bandgap; it is induced by an increase in the excitation density.

\subsection{Coupled coherent Bloch-phonon oscillations in superlattices}

The previous sections elucidated carrier-phonon interaction studied via coherent phonons at high excitation densities. An intriguing situation arises 

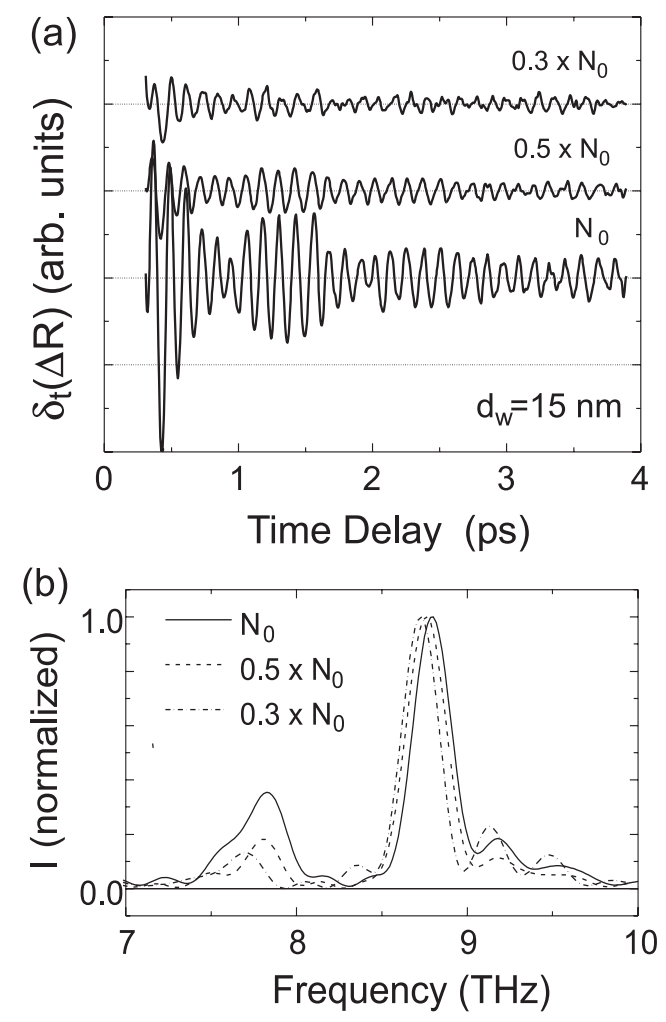

Fig. 15. (a) Oscillatory traces of electro-optic reflectivity changes of a $15 \mathrm{~nm}$ wide MQW sample at different excitation densities. The experiments are performed with a laser energy of $1.5 \mathrm{eV}$ at $300 \mathrm{~K}$. The maximum density $N_{0}$ corresponds to a bulk density of $3 \times 10^{18} \mathrm{~cm}^{-3}$. (b) Fourier transforms of the data in (a) normalized to the peak close to the LO phonon.

in quantum confined systems, when the electronic level spacing equals the LO phonon energy. In this case a large transition matrix element for phonon assisted relaxation is anticipated. The electronic level spacing in heterostructures can be manipulated by means of electric or magnetic fields. Inelastic light scattering experiments have been performed on Landau levels separated by an LO phonon energy, leading to the observation of magneto-polarons [77]. In semiconductor superlattices, the energy separation of Wannier-Stark levels can be tuned via an applied electric field over a range given by the electronic miniband width [78]. In this system, double and triple resonant Raman scattering experiments have been performed revealing increased scattering cross sections under resonance conditions [79, 80].

Semiconductor superlattices open the unique possibility to coherently excite electronic wavepackets with tunable oscillation frequencies, i.e. Bloch os- 


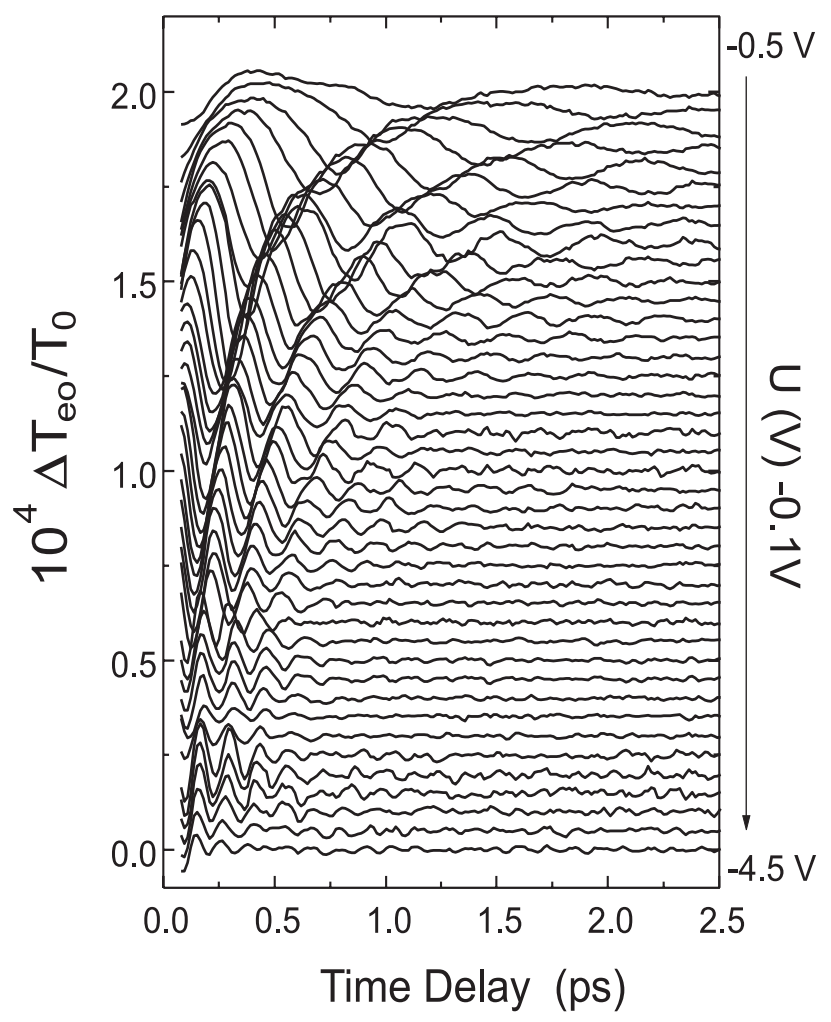

Fig. 16. Coherent Bloch oscillations extracted from anisotropic transmission changes in a 35 period $\mathrm{GaAs} / \mathrm{Al}_{0.3} \mathrm{Ga}_{0.7} \mathrm{As}$ superlattice of $67 \AA$ well width and $17 \AA$ barrier width. The calculated first electronic miniband width of the superlattice is $36 \mathrm{meV}$. The lattice temperature is $10 \mathrm{~K}$ and the excitation density is $3 \times 10^{9} \mathrm{~cm}^{-2}$. The data are depicted for different voltages applied to the superlattice that was embedded into a Schottky diode [31].

cillations, which are accessible by several experimental techniques $[81,82,83$, $84,85]$. The Bloch frequency is determined by $\nu_{B O}=e F d / h$, where $d$ is the superlattice period and $F$ the applied electric field. The Bloch wavepackets are associated with a macroscopic polarization oscillating along the growth direction of the superlattice, leading to the emission of $\mathrm{THz}$ radiation [83]. For details of Bloch oscillations we refer to the review by Kurz et al. [85].

Here we show results on coherent Bloch oscillations $\mathrm{GaAs} / \mathrm{Al}_{x} \mathrm{Ga}_{1-x} \mathrm{As}$ superlattices with large miniband widths, i.e. minibands with energetic widths equal or larger than the LO phonon energy, where the oscillation frequency can be tuned into resonance with the GaAs LO phonon. The detection is based on an optical anisotropy induced by the coherent electronic polarization [84], which is accomplished in basically the same experimental set-up as 
the electro-optic detection of coherent LO phonons in GaAs, but in transmission geometry.

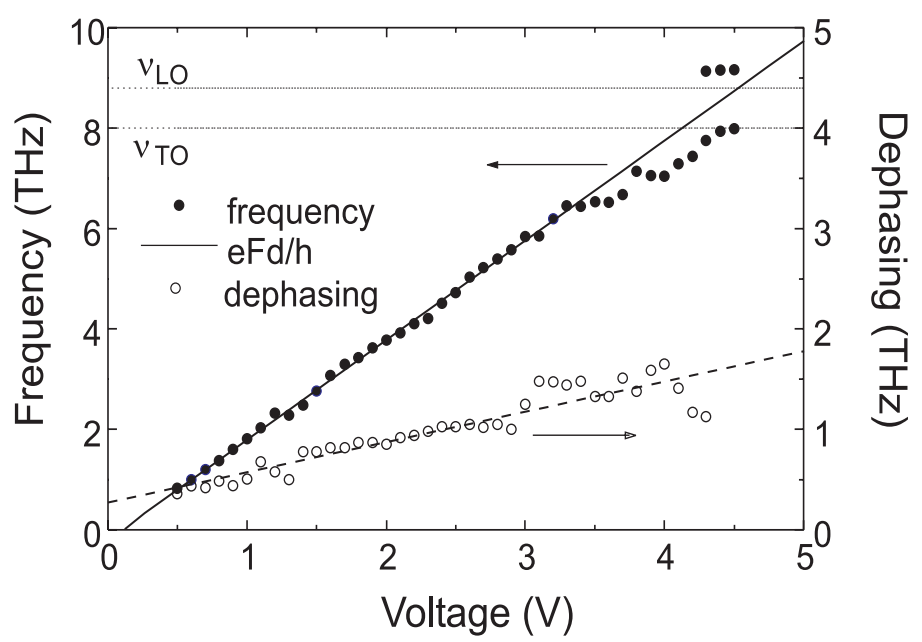

Fig. 17. Frequency and dephasing rate of Bloch oscillations depicted in the previous picture. The linear slope of the frequency obeys the Bloch relation $\nu=e F d / h[31]$.

Figure 16 depicts the extracted Bloch oscillations detected at $10 \mathrm{~K}$ for different voltages applied to the sample. The excitation density corresponds to $3 \times 10^{9}$ electron-hole pairs per $\mathrm{cm}^{2}$, which is more than 3 orders of magnitude lower than the densities used for the excitation of coherent LO phonons in GaAs via surface field screening. An increase in frequency can be clearly observed, from some $100 \mathrm{GHz}$ close to $10 \mathrm{THz}$, which is approximately the limit given by the miniband width. Beside the rise in frequency, a decrease in oscillation amplitude is observed which is due to the increased Stark localization of the wavepackets at increasing fields [86]. The effect of the reduced localization length and the associated decreased Bloch amplitude has been studied recently in $\mathrm{THz}$ emission [87] and more precisely in four-wave mixing experiments [88].

The change of oscillation frequency can be derived from the Fourier transforms of the time-resolved data in Fig. 16 and is depicted in Fig. 17. The internal electric field in the sample is proportional to the applied voltage plus an off-set voltage. The frequency follows over a wide range of voltages the linear relation expected for Bloch oscillations. Only for frequencies above $7 \mathrm{THz}$ the frequencies start to deviate slightly from this relation. For higher voltages, a splitting into a lower and upper branch at the TO and LO phonon frequencies of GaAs, respectively, is observed. The origin of this splitting is presently unknown, since at these densities the LO phonon cannot be screened. Non- 
linear interaction may account for the opening of a gap between the TO and LO phonon [89]. In addition to the deviations of the linear field-frequency relation, a decrease in the dephasing rate of about $30 \%$ is observed close to the phonon resonance (Fig. 17). This behavior is counterintuitive to an anticipated increased dephasing via resonant emission of LO phonons between Wannier-Stark levels, and would imply a stabilization of the electronic coherence via coupling to the lattice polarization. This behavior is in close analogy to the frequency dependence of the plasmon-phonon dephasing above resonance, when the lower-branch oscillation becomes more lattice like. It is important to note that this coupling observed in the superlattice occurs at 2 orders of magnitude lower carrier densities than necessary in bulk GaAs to tune the plasmon frequency into resonance with the LO phonon.
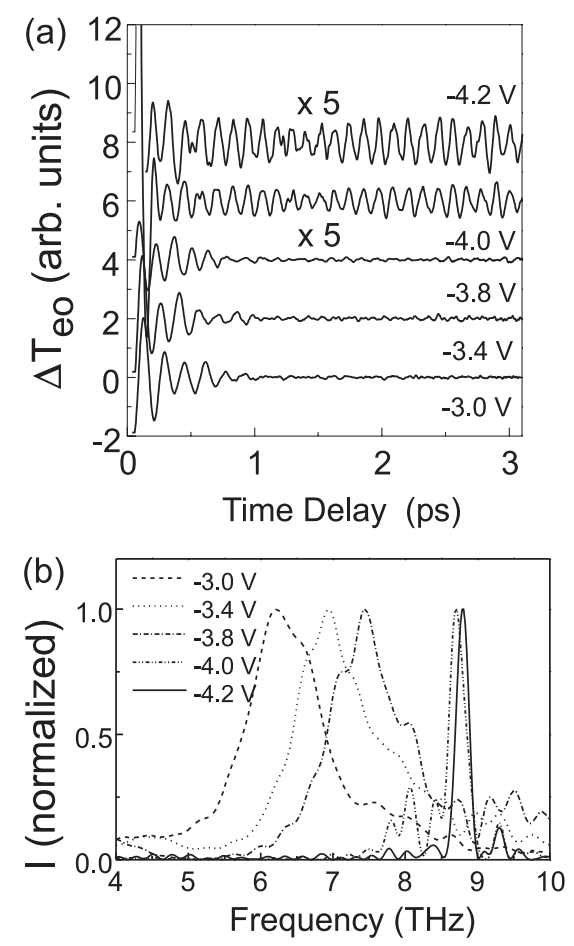

Fig. 18. (a) Time-resolved Bloch-phonon oscillations in a $\mathrm{GaAs} / \mathrm{Al}_{0.3} \mathrm{Ga}_{0.7} \mathrm{As}$ superlattice at $10 \mathrm{~K}$ for different applied reverse bias voltages. The data at the highest voltages are enlarged by a factor of 5 . (b) Normalized Fourier spectra of the data. From Ref. [31].

If the excitation density is increased by a factor of 3 , we observe that the coherent electronic polarization launches coherent phonons in the superlat- 
tice. This excitation process is described by the last term in Eqn. (4). Figure 18 depicts the oscillatory traces of Bloch-phonon oscillations in the same superlattice as investigated before. At reverse bias voltages below $-3.8 \mathrm{~V}$, the oscillations are pure electronic with dephasing times in the sub-picosecond range. When the applied voltage is increased by $-0.2 \mathrm{~V}$, the fast dephasing Bloch oscillations transit into a long living oscillation at the phonon frequency. The frequency of this oscillations matches the LO phonon frequency of bulk GaAs. This observation demonstrates that via Bloch-phonon coupling coherent phonons can be excited at densities which are more than two orders of magnitude smaller than the densities necessary to drive coherent LO phonons via field screening in bulk GaAs. This intriguing subject is under further experimental and theoretical investigation.

\subsection{Coherent acoustic phonons in superlattices}

The understanding of low-frequency excitations in semiconductors is of paramount importance for a complete picture of scattering and dephasing processes. The detailed knowledge of the lifetimes of acoustic phonons is important for description of heat dissipation in semiconductor devices. The investigations discussed so far deal with the excitation of coherent optical phonons. Here we present recent developments in the observation of coherent acoustic phonons in GaAs/AlAs based heterostructures (for further developments in low dimensional systems see also Sec. 8). The acoustic phonon branch is directly accessible by means of Brillouin scattering.

In semiconductor superlattices the effect of zone-folding leads to a series of acoustic phonons with $\omega \neq 0$ for $\mathbf{k}=0$ [90]. Zone folded acoustic phonons have been intensively studied throughout the last years by Raman scattering $[91,92]$. The first time-resolved observation of coherent acoustic phonons succeeded in GaAs/AlAs superlattices under resonant excitation of the first interband transitions [93]. The samples consist of 15 (18) monolayers GaAs wells and AlAs barriers. The coherent acoustic modes induce a change in the reflectivity, which is detected by the measurement of the time-derivative of the reflectivity change [94]. This technique is applied since the reflectivity change induced by the electronic excitation is much larger than the phonon induced oscillatory component. The data suggested that a stress in the wells is generated by the optical excitation, thus leading to the excitation of a mode of $\mathrm{B}_{2}$ symmetry within the $\mathrm{D}_{2 \mathrm{~d}}$ point group of the superlattice. However, this observation was in disagreement with Raman selection rules, i.e. the $\mathrm{B}_{2}$ mode is not Raman active.

More recently we [95] and other groups [96, 97] investigated the generation of coherent acoustic phonons in GaA/AlAs superlattices in more detail. Figure 19 shows high resolution time-resolved reflectivity changes obtained by excitation of a 19 monolayers GaAs/19 monolayers AlAs superlattice in resonance with the first interband transition. The reflectivity changes exhibit a complicated oscillatory structure in the time domain which stems from the 

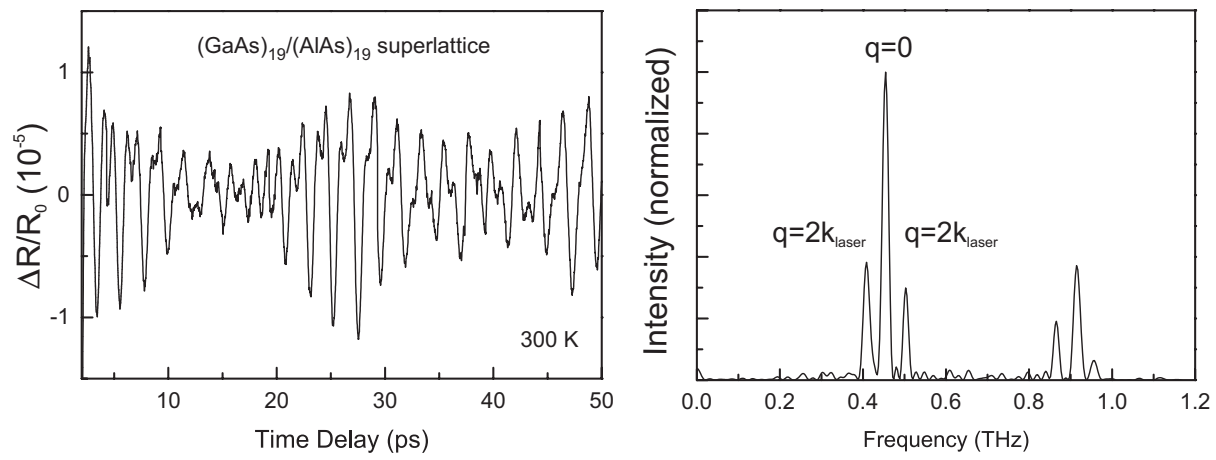

Fig. 19. Time-resolved reflectivity changes from a 19 monolayers GaAs/19 monolayers AlAs superlattice at room temperature (left). Fourier transform of the time resolved data (right, adapted from Ref. [95]).

superposition of 6 different frequencies. The reflectivity change of the order $10^{-3} \Delta R / R_{0}$, which is induced by the electronic excitation of the superlattice, has been subtracted. The observed modes belong to two triplets of the first and second order backfolded acoustic phonon spectrum. Each triplet has a strong center mode which corresponds to the $\mathbf{k}=0$ mode of $A_{1}$ symmetry. The two smaller peaks exactly match the calculated frequencies of phonons with a wavevector of $\mathbf{k}=2 \mathbf{k}_{\text {laser }}$. This observation points towards ISRS in forward $(\mathbf{k}=0)$ and backward scattering direction $\left(\mathbf{k}=2 \mathbf{k}_{\text {laser }}\right)$ as the driving force [95]. The detection process is observed to be resonantly enhanced at the lowest interband transitions and is assumed to be based on the energy shift of the interband transitions coupled to the acoustic mode via the deformation potential. The dephasing times of the acoustic modes are in the range of 100 ps. The linewidths in the Fourier spectrum in Fig. 19 are still limited by the finite time window of the experiment. By taking time scans over time delays $>200$ ps we recently obtained linewidths of $0.3 \mathrm{GHz}\left(<0.01 \mathrm{~cm}^{-1}\right)$ which opens the pathway for high resolution spectroscopy in this intriguing material system.

Coherent acoustic phonons have also been observed in superlattices of the Fibonacci sequence [98]. In this system, the quasi-periodicity of the superlattice generates a self-similar spectrum of phonon modes, which have also been observed in Raman spectroscopy [99].

\section{Coherent phonons in Tellurium}

\subsection{Selection rules for coherent phonon detection in Te}

For the case of bulk GaAs we illustrated in Sec. 4, how different nonlinear contributions to the coherent phonon detection process can be separated. Here 
we like to show, for Te single crystals, that different phonon modes can be separated on the base of the symmetry of the relevant Raman tensors. Tellurium is a perfect candidate for this purpose, since its crystal symmetry results in a set of only Raman-active, Raman- and IR-active, and only IR-active lattice modes. The phonon spectrum consists of 6 optical phonon modes. Te crystallizes in a hexagonal lattice (space group $D_{3}^{4}$ or $D_{3}^{6}$ ) containing three atoms per unit cell arranged in a helix along the c-axis. The lattice vibrations consist of a fully symmetrical, only Raman-active $\mathrm{A}_{1}$ mode $(3.6 \mathrm{THz})$, two degenerate Raman- and IR-active E modes (E'Tо/LO: $2.76 / 3.09 \mathrm{THz}$, E" то/LO: $4.22 / 4.26 \mathrm{THz})$ and one only IR-active $\mathrm{A}_{2}$ mode $\left(\mathrm{A}_{2, \mathrm{TO} / \mathrm{LO}}: 2.6 /-\right.$ $2.82 \mathrm{THz}$ )[100]. The internal polarization is either perpendicular (E modes) or parallel $\left(\mathrm{A}_{2}\right.$ mode $)$ to the c-axis. This will be relevant in the next section for the detection of $\mathrm{THz}$ emission.

The fully symmetric $A_{1}$ mode in Te is driven by the DECP mechanism, as has been demonstrated experimentally and confirmed theoretically [20]. However, the excitation of other modes of less symmetry cannot be explained within the DECP model, but is rather based on ISRS.

We applied to Te the same simultaneous detection of isotropic and anisotropic reflectivity changes as it has been already introduced for the electrooptic detection of LO phonons in GaAs in Sec.4.1. Again, the term isotropic is used to express that the phonon induced reflectivity changes do not depend on the relative angle between the probe pulse polarization and a certain crystal axis, while anisotropic means that two orthogonal components of the reflected probe pulse are subtracted from each other. This notation should be clearly distinguished from the Raman notation of polarized and depolarized spectra.

Figure 20 depicts the time-resolved isotropic and anisotropic reflectivity changes recorded in Te at a surface perpendicular to the c-axis. The experiments are performed with a CPM dye laser. The isotropic reflectivity shows a strong modulation at the frequency of the $\mathrm{A}_{1}$ mode, while the anisotropic reflectivity exhibits a more complicated oscillatory behavior. The Fourier transformed time-domain data reveal the two $\mathrm{E}$ modes and the absence of the $\mathrm{A}_{1}$ mode in the anisotropic reflectivity change.

The manifestation of phonon modes in the anisotropic reflectivity changes results from the off-diagonal elements of the Raman tensor. The anisotropic reflectivity can be expressed as

$$
\Delta R_{j}-\Delta R_{k} \sim \mathbf{E}_{j}^{r}(\partial \chi / \partial Q) \mathbf{E}^{i} Q-\mathbf{E}_{k}^{r}(\partial \chi / \partial Q) \mathbf{E}^{i} Q,
$$

where $\mathbf{E}^{i}$ and $\mathbf{E}^{r}$ are the incident and reflected probe fields, respectively. $(\partial \chi / \partial Q)$ denotes the Raman tensor, which contains the following non-zero elements for the two doubly degenerate E modes [100]:

$$
\begin{aligned}
E(x): \quad(\partial \chi / \partial Q)_{x x}=-(\partial \chi / \partial Q)_{y y}=c \\
(\partial \chi / \partial Q)_{y z}=(\partial \chi / \partial Q)_{z y}=d
\end{aligned}
$$



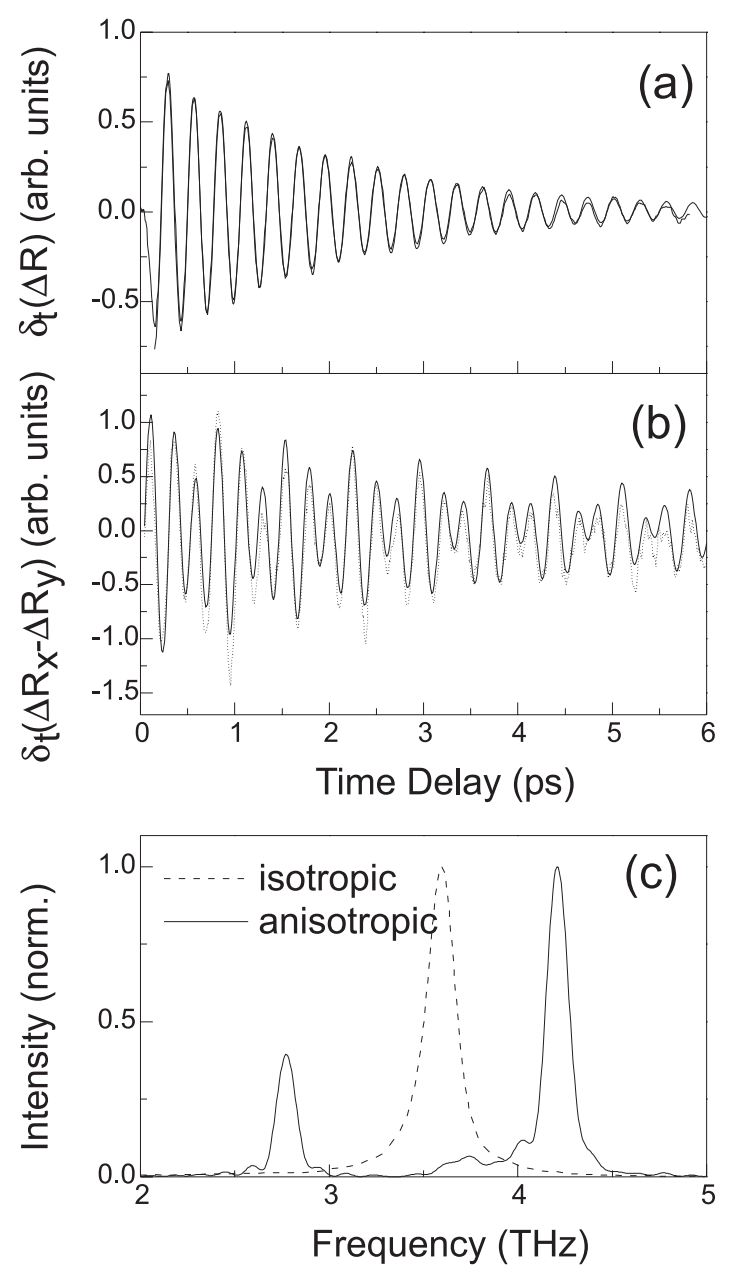

Fig. 20. Oscillatory component of the (a) isotropic and (b) anisotropic reflectivity change from single crystal Te, surface perpendicular to the c-axis, obtained at 2 $\mathrm{eV}$. The data are recorded in a time-differential method [94]. Numerical fits (thin lines) are hard to distinguish from the experimental data. (c) Fourier transform of the time-resolved signals.

$$
\begin{aligned}
E(y):(\partial \chi / \partial Q)_{x y} & =(\partial \chi / \partial Q)_{y x}=-c \\
(\partial \chi / \partial Q)_{x z} & =(\partial \chi / \partial Q)_{z x}=-d
\end{aligned}
$$

The Raman tensor of the $\mathrm{A}_{1}$ mode contains diagonal elements only. For the $\mathrm{c} \perp$ surface, we obtain the anisotropic reflectivity change induced by the E modes: $\Delta R_{x}-\Delta R_{y} \sim \sqrt{2} c\left(Q_{E(x)}+Q_{E(y)}\right)$, i.e. a non-zero contribution from the coherent displacement amplitudes of the degenerate E modes. For 
the fully symmetric $\mathrm{A}_{1}$ mode, $\Delta R_{x}-\Delta R_{y}$ is zero. These considerations are confirmed by the experiments illustrated in Fig.20.

The detection of modes other than those of $\mathrm{A}_{1}$ symmetry in (anisotropic) reflectivity changes has been recently confirmed by Hase et al. for the case of $\mathrm{Bi}$ [37] and Garrett et al. for Sb [36]. In transparent media Raman selection rules in the phonon detection process have been observed by Liu at al. in $\mathrm{LaAlO}_{3}[101]$.

\subsection{THz emission from coherent phonons}

In the previous section the relevance of Raman processes for the phonon detection has been established. Here we discuss the detection of IR-active but Raman inactive phonons. Since the atomic displacements associated with these modes do not lead to changes in the optical properties in the visible range of the spectrum, they can be neither excited nor detected via Raman processes. By means of time-resolving the $\mathrm{THz}$ emission from semiconductors following pulsed excitation, an emission at the phonon frequency is expected. The $\mathrm{THz}$ emission from coherent phonons has been first theoretically proposed for coherent (Raman and IR active) LO phonons in GaAs by Kuznetsov and Stanton [9]. However, the LO phonon frequency of GaAs is not within the detection bandwidth of conventional dipole antennas used for time-resolved $\mathrm{THz}$ detection. Therefore we have chosen to investigate the $\mathrm{THz}$ emission from single crystal Te, which exhibits IR-active phonons in a lower frequency range.

In the THz-emission experiments, the sample is excited under $45^{\circ}$ incidence by Ti:sapphire laser pulses with $1.75 \mathrm{eV}$ photon energy and a pulse duration of $150 \mathrm{fs}$. The coherent $\mathrm{THz}$ radiation emitted in the direction of the reflected optical beam is collected with two paraboloidal mirrors and detected with a submillimeter dipole antenna that is gated by a second timedelayed laser pulse. The current in the dipole antenna, which is proportional to the incident electric field, is recorded as a function of the time delay [40]. The sensitivity of the detection system peaks at about $1 \mathrm{THz}$ and extends up to 3 to $4 \mathrm{THz}[40]$.

Figure 21 (a) shows the measured electric field emitted from a surface perpendicula to the c-axis. The data are compared to the emission spectrum of a broad-band emitter ( $\mathrm{InP}$ ), where the emission is based on the screening of the surface field [40]. The signal from Te consists of a strong initial emission followed by a periodically modulated tail. The strong peak is attributed to a polarization that results from the ultrafast build-up of an electric photoDember field [29], which is driven by the strong carrier gradient at the surface and the difference in electron and hole diffusion coefficients [30]. This effect is amplified by differences in the transient electron and hole temperatures. Numerical simulations confirm a Dember field build-up with amplitudes of $50 \mathrm{kV} / \mathrm{cm}$ and a rise-time of $100 \mathrm{fs}$. The screening of depletion-layer fields as the source of the radiation can be neglected in Te, because of a low density of 

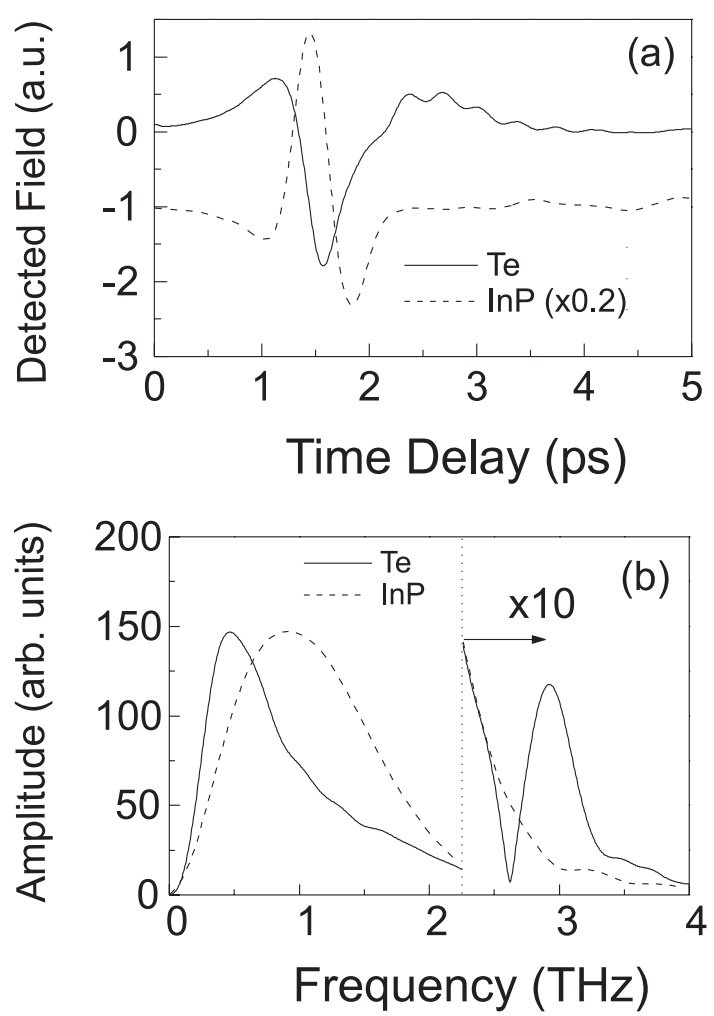

Fig. 21. (a) THz emission from Te (surface perpendicular to the c-axis) and InP. (b) Fourier transform of the time domain data in (a). The high frequency part is enlarged by a factor of 10. Adapted from Ref. [10].

charged surface states within the small bandgap [102]. The amplitude of the initial THz signal due to the Dember field is only a factor of 5 weaker than the emission from a polar semiconductor with strong surface fields (e.g. InP) for the same excitation power. Figure 21 (b) depicts the amplitude of the Fourier transform of the time-domain signal. The frequency spectrum reveals, for Te, a broad peak at approximately $500 \mathrm{GHz}$. At high frequencies, the signal decreases to nearly zero at $2.62 \mathrm{THz}$ before reaching a second maximum at about $2.9 \mathrm{THz}$. For clarity, this part of the spectrum is enlarged by a factor of 10. The high-frequency spectrum of a broadband emitting surface (InP, also $\times 10$, not normalized) representing the antenna response is shown for comparison. By polarization analysis of the high frequency emission it can be shown that the emission stems from the coherently excited $\mathrm{A}_{2}$ mode at $2.82 \mathrm{THz}$, which is only IR active. This mode has an internal polarization parallel to the c-axis, i.e. perpendicular to the excited surface. Thus this mode couples effectively to the Dember field. 
The excitation mechanism via the build-up of a Dember field is confirmed by numerical calculations of the non-equilibrium electron-hole distribution close to the surface [30]. The Dember field perpendicular to the sample surface has a transverse wavevector due to the $45^{\circ}$ incidence of the optical pulse. According to the phonon-polariton dispersion for transverse electromagnetic waves, the polarization and the electric field inside the sample will dominantly oscillate at the LO phonon frequency at small wavevectors, while the polarization at the TO frequency is small. As a result, the $\mathrm{THz}$ emission is much stronger at the LO frequency than at the TO frequency. The spectral shape of the $\mathrm{THz}$ emission is furthermore influenced by the frequency dependence of the outcoupling efficiency. The frequency dependence of the refractive index makes the outcoupling most efficient at the LO frequency and least efficient at that of the TO phonons. This effect leads to a further increase in intensity at the LO frequency and a decrease at the TO frequency. In addition, plasmon-phonon coupling as discussed in Sec. 4.2, is expected to change the spectral shape of the $\mathrm{THz}$ emission [11]. However, due to the large carrier gradient close to the surface, the plasma frequency is not well defined. Recently, the results summarized here have been experimentally confirmed by Tani at al. in Te, PbTe and CdTe [12].

\subsection{Impulsive mode softening of phonons}

One important aspect of phonon spectroscopy is the fact that the timeresolved detection of the phonon frequency provides information on the dynamics of phase transitions. The transition from a crystalline to an amorphous state, e.g., is accompanied by a strong change in the phonon spectrum. Information about the origin of optically induced phase transitions is essential for the optimization of material processing based on high power fs laser sources, as well for future phase-change optical recording materials. One specific form of phase transitions proposed for excitation with fs laser pulses is non-thermal melting, where the crystal is destabilized by the optical excitation before energy transfer from the excited carriers to the lattice can take place. Several investigations deal with this problem. Most of them are based on time-resolved second harmonic generation (SHG), which vanishes when the crystal loses its symmetry. It has been reported, that this process occurs on time scales which are shorter than the typical energy transfer times from the electronic system to the lattice [103, 104, 105]. Theoretically it has been predicted that a non-thermal phase transition may occur when more than $9 \%$ of all valence electrons are excited into antibonding conduction band states [106, 107].

For an investigation of this intriguing phenomenon, we investigate the coherent phonon dynamics in single crystal Te excited by amplified CPM laser pulses of $2 \mathrm{eV}$ photon energy [108]. This laser system delivers pulse energies of $1.2 \mu \mathrm{J}$ at a repetition rate of $6.8 \mathrm{kHz}$. Figure 22 shows the time-resolved reflectivity changes at different pump fluences. The reflectivity changes are 
(a)

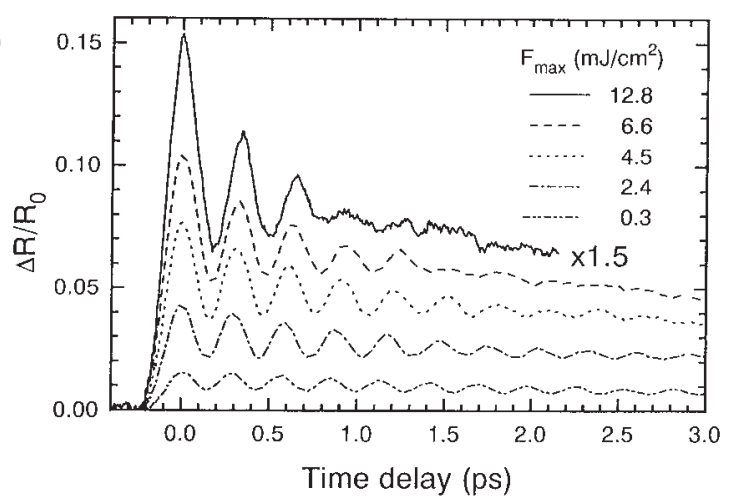

(b)

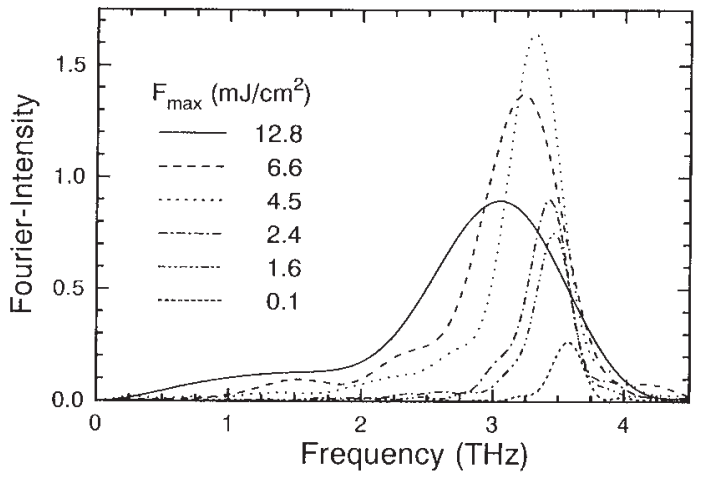

Fig. 22. (a) Time-resolved reflectivity changes from single crystal Te for different excitation fluences as given in the figure. (b) Not normalized Fourier transforms of the data in (a) (adapted from Ref. [108]).

dominated by the $A_{1}$ mode excited via DECP as has already been shown in Sec. 6.1. The oscillation frequency decreases linearly for this range of fluences from 3.6 THz, i.e. the value of the unperturbed phonon resonance, to approximately $3 \mathrm{THz}$. A fit to the data shows that this mode softening is instantaneous. This is a clear hint for non-thermal melting of the crystal, since the energy transfer to the lattice is expected to take place on a longer time-scale. A numerical simulation of the phonon dynamics, including the effect of carrier diffusion in this time scale, gives an estimate for the critical excitation density necessary for the non-thermal melting of the crystal, which is about $20 \%$ of all valence band electrons [109]. A careful comparison of the reflectivity change induced by the coherent phonons with the reflectivity changes induced by heating the crystal allows one to determine the relative lattice displacement of $0.36 \%$ of the equilibrium lattice constant for the highest excitation density in Fig. 22 [110]. 
In $\mathrm{Ti}_{2} \mathrm{O}_{3}$ a transient shift of the phonon frequency, similar to the observations in Te, has been observed by Cheng at al. [111]. The Raman-active $\mathrm{A}_{1} g$ mode of $\mathrm{Ti}_{2} \mathrm{O}_{3}$ at $7 \mathrm{THz}$ is excited via the DECP mechanism. An estimation of the lattice displacement associated with the coherent atomic motion shows that the semiconductor-to-semimetal transition in this narrow-bandgap material is modulated at $7 \mathrm{THz}$. These experiments demonstrate the feasibility to control material properties on a subpicosecond time scale via the excitation of large vibrational amplitudes.

\section{Coherent phonons in other materials}

\subsection{Coherent phonons in high-temperature superconductors}

The role of electron-phonon coupling in the mechanism responsible for high temperature superconductivity (HTSC) is of central importance for the understanding of this phenomenon. Phonon modes in HTSC's have been extensively investigated by Raman spectroscopy, for a review we refer to Ref. [112]. The study of coherent phonon dynamics may contribute important information, since the dynamics of Cooper pairs and quasi particles can be observed concomitantly with the lattice dynamics on a sub-ps time scale.

The first observation of coherent phonons in HTSC materials has been reported by Chwalek et al. [113] in the non-superconducting phase and Albrecht et al. [114] for both superconducting and non-superconducting phases. As intriguing question in these materials concerns the excitation mechanism for coherent phonons - especially in the superconducting phase - and the relation to the optically induced breaking of Cooper pairs.

Figure 23 depicts the oscillatory contributions to time resolved reflectivity changes of $\mathrm{YBa}_{2} \mathrm{Cu}_{3} \mathrm{O}_{7-\delta}$ (YBCO) in the superconducting phase [115]. The oscillations consist of a superposition of the $\mathrm{A}_{1 g}$ modes of $\mathrm{Ba}$ and $\mathrm{Cu}(2)$ in the $\mathrm{CuO}_{2}$ plane, with frequencies of $3.6 \mathrm{THz}$ and $4.5 \mathrm{THz}$, respectively [116]. The amplitudes, phases and dephasing times are obtained with high accuracy from these fits. Figure 24 depicts the coherent phonon amplitudes obtained from time-resolved reflectivity changes in YBCO measured at different lattice temperatures. While there is a weak temperature dependence of the amplitude of the coherently excited $\mathrm{A}_{1 g}$ mode of $\mathrm{Ba}$ and $\mathrm{Cu}(2)$ above $\mathrm{T}_{c}$, the amplitude of the Ba mode strongly increases below $\mathrm{T}_{c}$. In addition the phase of this mode changes strongly as a function of temperature, indicating a different excitation mechanism above and below $\mathrm{T}_{c}$ to be responsible for the coherent phonon generation. The amplitude dependence is well reproduced by a two-fluid model below $\mathrm{T}_{c}[114,115]$, indicating a strong correlation between the coherent phonon amplitude and the perturbation of the electronic system. Above $\mathrm{T}_{c}$ both modes are excited via DECP, leading to an impulsive bond-weakening of the lattice. Below $\mathrm{T}_{c}$ the excitation mechanism for the Ba mode can be explained by the ultrafast breaking of Cooper pairs induced by the optical pulse. The pair breaking strongly changes the conductivity in 


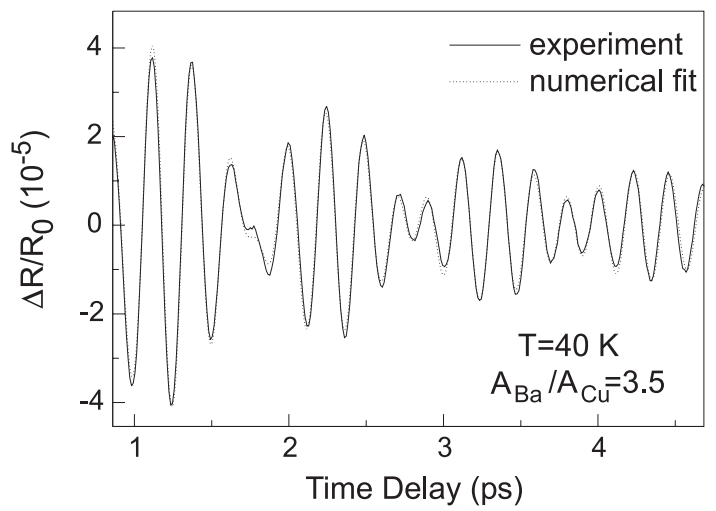

Fig. 23. Oscillatory contribution of time-resolved reflectivity change (solid line) of a $\mathrm{YBa}_{2} \mathrm{Cu}_{3} \mathrm{O}_{7-\delta}$ thin film. The experiments are performed with a Ti:sapphire laser at $1.55 \mathrm{eV}$. The numerical fit is based on two sine functions with the Ba-mode frequency $(3.6 \mathrm{THz})$ and the $\mathrm{Cu}$-mode frequency (4.5 THz). From Ref. [115].

the $\mathrm{CuO}_{2}$ planes. This produces a sudden change in the local crystal field of the $\mathrm{Ba}$ atoms, leading to their coherent displacement. The $\mathrm{Cu}(2)$ mode is less sensitive to the local crystal field changes. Similar changes of intensities of the $\mathrm{Ba}$ and $\mathrm{Cu}(2)$ observed in the time-resolved phonon amplitudes have been also observed in the ordinary Raman spectra [117]. The relevance of the sudden screening of the local crystal field and the implications on the generation of coherent phonons in HTSC have been confirmed recently in a systematic study on differently doped $\mathrm{YBCO}$ and in $\mathrm{Bi}_{2} \mathrm{Sr}_{2} \mathrm{CaCu}_{2} \mathrm{O}_{8}$ [115].

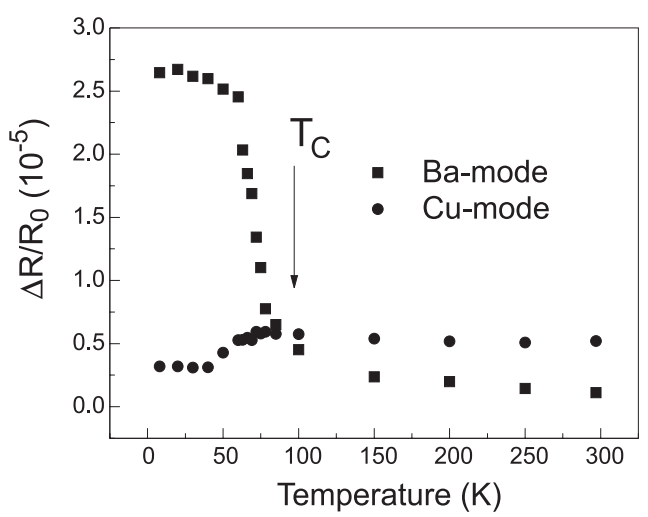

Fig. 24. Coherent phonon amplitude of the $\mathrm{Ba}$ and $\mathrm{Cu}(2)$ modes in $\mathrm{YBa}_{2} \mathrm{Cu}_{3} \mathrm{O}_{7-\delta}$ as a function of lattice temperature. From Ref. [115] 


\subsection{Coherent phonon-polaritons in ferroelectric crystals}

In Sec. (6.2) we discussed the coherent excitation of an only IR active phonon mode via an electric Dember field and the associated emission of $\mathrm{THz}$ radiation. There exists a more direct way to generate FIR light inside a crystal via ISRS $[118,119]$, difference frequency mixing $[120,121]$ or the optical Cerenkov effect [122]. In the generation mechanism based on difference frequency mixing two laser fields $E_{k}$ and $E_{l}$ provided by two fs laser pulses with spatial and temporal overlap are focused on the same spot on the sample [121]. This process is based on the second order nonlinear susceptibility $\chi^{(2)}$ of the crystal. By choosing a certain angle between the two laser pulses a well defined mode on the phonon-polariton dispersion can be selected corresponding to the wave vector $\mathbf{k}=\mathbf{k}_{k}-\mathbf{k}_{l}$. This method creates two counterpropagating polaritons within the laser spot. The generated coherent phonon-polariton can be probed via a third time-delayed laser pulse positioned closely on either side of the excitation spot. The polariton may be detected by probe light diffracted via the electro-optic effect. Since the difference frequency mixing is performed with laser energies well below interband resonances, high peak intensities are necessary for these experiments. Therefore the experiments were performed with amplified laser pulses, e.g. with an amplified CPM laser at a photon energy of $2 \mathrm{eV}, 60 \mathrm{fs}$ pulse duration and a pulse energy of $\sim \mu \mathrm{J}$.

By this technique, Bakker et al. were able to map out the phonon-polariton dispersion with high sensitivity in the low frequency range in several ferroelectric crystals $\left(\mathrm{LiTaO}_{3}[121]\right.$ and $\left.\mathrm{LiNbO}_{3}[123]\right)$. Due to the high sensitivity of this method, new resonances in the dispersion could be detected at 0.95 $\mathrm{THz}$ in $\mathrm{LiTaO}_{3}$ and at $1.3 \mathrm{THz}, 2.4 \mathrm{THz}, 3.4 \mathrm{THz}$ and $4.1 \mathrm{THz}$ in $\mathrm{LiNbO}_{3}$. These frequencies, and the corresponding temperature dependence of the dephasing times, allowed the authors to develop a quantum mechanical model for the ferroelectric phase transition. The newly observed resonances turned out to be quantum mechanical tunneling resonances of the lowest phonon mode. A recent review on this subject can be found in Ref. [124].

Furthermore, time-resolved phonon-polariton studies have been performed by Vallée and Flytzanis [125], where phonon-polariton packets are generated in one crystal and the detection is accomplished in a second crystal separated by an air gap from the first. This technique allows the study of the influence of interfaces and surfaces on the phonon-polariton propagation.

\section{Recent developments}

There exist several interesting recent reports on the observation of coherent phonons in a variety of materials, which we like to summarize here.

The application of laser pulses of $10 \mathrm{fs}$ to $20 \mathrm{fs}$ duration directly derived from a resonator enables one to time-resolve phonon oscillations in the range beyond $10 \mathrm{THz}$. Fleischer et al. recently demonstrated the observation of a coherently excited $A_{g}(1)$ mode at $14.9 \mathrm{THz}$ in doped $\mathrm{C}_{60}$ molecules [126]. In 
addition to this mode, a low frequency mode around $4.5 \mathrm{THz}$ was observed which could not be observed in CW Raman experiments. These modes are attributed to arise from the dopant atoms.

Hase at al. recently observed coherent plasmon-phonon modes also from the upper branch of the coupled mode dispersion by applying $20 \mathrm{fs}$ laser pulses [127]. These findings confirm that the frequency of coherent modes is given by both, the optically excited carriers and the carriers from the background doping of the sample [57].

Coherent optical and acoustic modes have been observed recently in semiconductor quantum dots $[128,129,130,131,132]$. Resonant excitation of quantum dots in conjunction with strong electron-phonon coupling leads to a modulation of a four wave mixing signal with the frequency of the LO phonon in CdSe quantum dots $[128,129]$ and of the LO and TO mode in InP quantum dots [131]. Coherent acoustic modes could be observed in resonant pump-probe experiments on $\mathrm{PbS}$ quantum dots [132, 133]. The generation mechanism is based on deformation-potential coupling of the lowest-order spheroidal acoustic mode to the quantum-dot excitons [132]. The dominant damping mechanism has benn identified as radiative loss from the quantum dots to the surrounding glass [133]. In contrast to the work on CdSe and InP quantum dots, the optical mode could not be observed in $\mathrm{PbTe}$ quantum dots, suggesting differences in the exciton-phonon coupling strength in quantum dots of IV-VI, II-VI and III-V composition.

One basic concept of quantum mechanics is Heisenberg's uncertainty principle for two non-commuting observables. However, the quantum fluctuations of one observable may be reduced below the vacuum limit at the expenses of the other such that the product of the fluctuations still obeys the uncertainty relation. A state prepared in this way is denoted as vacuum squeezed state and has first been observed by Slusher et al. for photons [134]. Coherent phonons and polaritons may provide the access to the realization of squeezed states in solids [135, 136]. Recently, Garrett et al. achieved the observation of phonon squeezed states in $\mathrm{KTaO}_{3}$ [35]. The excitation is based on a second-order process leading to an impulsive change of the phonon frequency of transverse acoustic modes. The squeezing factor derived from the observation of coherently excited TA modes is in the range of $10^{-6}$, which is still small compared to values obtained in squeezing experiments with photons. These findings on phonon squeezed states in solids are supported by experiments in $\mathrm{SrTiO}_{3}$ by the same group [137].

The coherent control of electron-LO-phonon scattering has been achieved in fs four-wave mixing experiments, where the delay of two excitation pulses has been varied on a time scale of several tens of femtoseconds before electronLO-phonon scattering is completed [138]. These experiments allowed the control of the scattering dynamics in a coherent fashion, thus leading to the suppression or enhancement of the scattering process within the time in which the process is not yet fully accomplished. 


\section{Conclusion}

In this chapter we presented an overview of the feasibilities of time-resolved detection of coherently excited lattice vibrations in the $\mathrm{THz}$ frequency range. In contrast to conventional Raman scattering, the generation and detection processes of coherent phonons can be well separated leading to interesting insights into the nonlinear interaction between sub-ps light pulses and semiconductors, superconductors and ferroelectric crystals, etc. In recent years, coherent phonon spectroscopy has revealed many results important for the understanding of lattice dynamics and nonlinear phonon-photon interaction. This development was strongly driven by the improvement of sub-picosecond laser sources. Most recent developments of stable laser sources with pulse durations in the range of $10 \mathrm{fs}$ and high peak powers up to $\mu \mathrm{J}$ per pulse in combination with pulse shaping techniques should allow us to perform a huge variety of intriguing experiments on coherent phonon dynamics, including the coherent control over the lattice motion up to enormously large displacements. In comparison to $\mathrm{CW}$ inelastic light scattering experiment the field of time-resolved coherent phonon spectroscopy is relatively young. The spreading and availability of stable femtosecond lasers in all ranges of wavelengths and energies will certainly intensify the research in this field.

\section{Acknowledgment}

The authors thank the following people for their valuable contributions to this work: H. J. Bakker, A. Bartels, G. Bauer, M. Cardona, A. Förster, A. Ghosh, J. Geurts, P. Grosse, S. Hunsche, C. Jaekel, A. M. T. Kim, K. Köhler, W. Kütt, A. V. Kuznetsov, K. Mizoguchi, S. Nakashima, M. Nakayama, T. Pfeifer, H. G. Roskos, R. Scholz, V. Wagner, and T. Pfeifer. We gratefully acknowledge support by the Deutsche Forschungsgemeinschaft and the Volkswagen Stiftung.

\section{References}

1. J. Shah, Ultrafast Spectroscopy of Semiconductors and Semiconductor Nanostructure, Vol. 115 of Solid State Science (Springer, Berlin, 1996).

2. W. E. Bron, J. Kuhl, and B. K. Rhee, Phys. Rev. B 34, 6961 (1986).

3. D. von der Linde, in Ultrashort Laser Pulses, Vol. 60 of Topics Appl. Phys., edited by W. Kaiser (Springer, Berlin, 1988).

4. J. A. Kash and J. C. Tang, in Spectroscopy of Nonequilibrium Electrons and Phonons, edited by C. V. Shank and B. P. Zakharchenya (Elsevier, Amsterdam, 1992), pp. 113-168.

5. F. Vallée and F. Bogani, Phys. Rev. B 43, 12049 (1991).

6. F. Vallée, Phys. Rev. B 49, 2460 (1994).

7. F. Ganikhanov and F. Vallée, Phys. Rev. B 55, 15614 (1997). 
8. W. E. Bron, in Coherent Optical Interactions in Semiconductors, Vol. 330 of NATO ASI B, edited by R. T. Phillips (Plenum, New York, 1994), pp. 199222.

9. A. V. Kuznetsov and C. J. Stanton, Phys. Rev. Lett. 73, 3243 (1994).

10. T. Dekorsy, H. Auer, C. Waschke, H. J. Bakker, H. G. Roskos, H. Kurz, V. Wagner, and P. Grosse, Phys. Rev. Lett. 74, 738 (1995).

11. A. V. Kuznetsov and C. J. Stanton, Phys. Rev. B 51, 7555 (1995).

12. M. Tani, R. Fukasawa, H. Abe, S. Matsuura, K. Sakai, and S. Nakashima, J. Appl. Phys. 83, 2473 (1998).

13. G. C. Cho, W. Kütt, and H. Kurz, Phys. Rev. Lett. 65, 764 (1990).

14. G. C. Cho, H. J. Bakker, T. Dekorsy, and H. Kurz, Phys. Rev. B 53, 6904 (1996).

15. W. Kütt, in Festkörperprobleme/Advances in Solid States Physics, edited by U. Rössler (Vieweg, Braunschweig, 1992), Vol. 32, pp. 113-129.

16. A. Lauberau and W. Kaiser, Rev. Mod. Phys. 50, 607 (1978).

17. R. Merlin, Sol. Stat. Comm. 102, 207 (1997).

18. T. K. Cheng, S. D. Brorson, A. S. Kazeroonian, J. S. Moodera, G. Dresselhaus, M. S. Dresselhaus, and E. P. Ippen, Appl. Phys. Lett. 57, 1004 (1990).

19. T. K. Cheng, J. Vidal, M. J. Zeiger, G. Dresselhaus, M. S. Dresselhaus, and E. P. Ippen, Appl. Phys. Lett. 59, 1923 (1991).

20. H. J. Zeiger, J. Vidal, T. K. Cheng, E. P. Ippen, G. Dresselhaus, and M. Dresselhaus, Phys. Rev. B 45, 768 (1992).

21. Y.-X. Yan, E. B. Gamble, and K. A. Nelson, J. Chem. Phys. 83, 5391 (1985).

22. S. DeSilvestri, J. G. Fujimoto, E. B. Gamble, L. R. Williams, and K. A. Nelson, Chem. Phys. Lett. 116, 146 (1985).

23. Y.-X. Yan and K. A. Nelson, J. Chem. Phys. 86, 6240 (1987).

24. Y.-X. Yan and K. A. Nelson, J. Chem. Phys. 86, 6257 (1987).

25. J. Chesnoy and A. Mokthari, Phys. Rev. B 38, 3566 (1988).

26. T. Dekorsy, Ph.D. thesis, RWTH Aachen, 1997.

27. T. Pfeifer, T. Dekorsy, W. Kütt, and H. Kurz, Appl. Phys. A 55, 482 (1992).

28. T. Dekorsy, T. Pfeifer, W. Kütt, and H. Kurz, Phys. Rev. B 47, 3842 (1993).

29. H. Dember, Phys. Z. 32, 554 (1931).

30. T. Dekorsy, H. Auer, H. J. Bakker, H. G. Roskos, and H. Kurz, Phys. Rev. B 53, 4005 (1996).

31. T. Dekorsy, A. M. T. Kim, G. C. Cho, K. Köhler, and H. Kurz, in Ultrafast Phenomena X, edited by P. F. Barbara, J. G. Fujimoto, W. H. Knox, and W. Zinth (Springer, Berlin, 1996), pp. 382-384.

32. B. B. Varga, Phys. Rev. 137, 1896 (1965).

33. A. Mooradian and G. B. Wright, Phys. Rev. Lett. 16, 999 (1966).

34. A. Mooradian and A. L. McWorther, Phys. Rev. Lett. 19, 849 (1967).

35. G. A. Garrett, A. G. Rojo, A. K. Sood, J. F. Whitaker, and R. Merlin, Science 275, 1638 (1997).

36. G. A. Garrett, T. F. Albrecht, J. F. Whitaker, and R. Merlin, Phys. Rev. Lett. 77, 3661 (1996).

37. M. Hase, K. Mizoguchi, H. Harima, S. Nakashima, M. Tani, K. Sakai, and M. Hangyo, Appl. Phys. Lett. 69, 2474 (1996).

38. W. A. Kütt, W. Albrecht, and H. Kurz, IEEE J. Quant. Electr. QE 28, 2434 (1992). 
39. M. Kuball, N. Esser, T. Ruf, C. Ullrich, M. Cardona, K. Eberl, A. GarciaCristobal, and A. Cantarero, Phys. Rev. B 51, 7353 (1995).

40. X.-C. Zhang and D. H. Auston, J. Appl. Phys. 71, 326 (1992).

41. Q. Wu and X. C. Zhang, Appl. Phys. Lett. 70, 1784 (1997).

42. Q. Wu and X. C. Zhang, in Postdeadline paper at the Conference on Lasers and Electro Optics (Optical Society of America, Washington DC, 1997), pp. $1-3$.

43. A. Cantarero, C. Trallero-Giner, and M. Cardona, Phys. Rev. B 39, 8388 (1989).

44. A. Cantarero, C. Trallero-Giner, and M. Cardona, Phys. Rev. B 40, 12290 (1989).

45. M. J. Rosker, F. W. Wise, and C. L. Tang, Phys. Rev. Lett. 57, 321 (1986).

46. This system has become commercially available as a PC based system by GWU Lasertechnik, Germany.

47. S. M. Sze, Physics of Semiconductor Devices, 2nd ed. (J.Wiley \& Sons, New York, 1981).

48. A. Yarif, Introduction to Optical Electronics (Holt, Rinehart and Winston, New York, 1971).

49. L. Min and R. J. D. Miller, Appl. Phys. Lett. 56, 524 (1990).

50. M. Hase, K. Mizoguchi, H. Harima, S. Nakashima, and K. Sakai, Phys. Rev. B 58, 5448 (1998).

51. J. G. Gay, J. D. Dow, E. Burstein, and A. Pinzcuk, in Light Scattering in Solids, edited by M. Balkanski (Flammarion, Paris, 1971), pp. 33-38.

52. R. M. Martin and L. M. Falicov, in Light Scattering in Solids, Vol. 8 of Topics in Applied Physics, edited by M. Cardona (Springer, Berlin, 1975), pp. 79-145.

53. M. Cardona, in Light Scattering in Solids, Vol. 50 of Topics in Applied Physics, edited by M. Cardona (Springer, Berlin, 1982), pp. 19-178.

54. T. Pfeifer, T. Dekorsy, W. Kütt, and H. Kurz, in Phonon Scattering in Condensed Matter VII, Vol. 112 of Solid-State Sciences, edited by M. Meissner and R. O. Pohl (Springer, Berlin, 1993), p. 110.

55. P. C. Becker, H. L. Fragnito, C. Brito-Cruz, J. Shah, R. L. Fork, J. E. Cunningham, J. E. Henry, and C. V. Shank, Appl. Phys. Lett. 53, 2089 (1988).

56. C. C. Cho, Ph.D. thesis, RWTH Aachen, 1997.

57. G. C. Cho, T. Dekorsy, H. J. Bakker, R. Hövel, and H. Kurz, Phys. Rev. Lett. 77, 4062 (1996).

58. R. Kersting, K. Unterrainer, G. Strasser, H. Kaufmann, and E. Gornik, Phys. Rev. Lett. 79, 3038 (1997).

59. W. Sha, A. L. Smirl, and W. F. Tseng, Phys. Rev. Lett. 74, 4273 (1995).

60. W. de Jong, A. van Etteger, P. van Hall, and T. Rasing, Surface Science 377, 355 (1997).

61. Y. M. Chang, L. Xu, and H. W. K. Tom, in Ultrafast Phenomena X, Vol. 62 of Springer Series in Chemical Physics, edited by P. F. Barabara, J. G. Fujimoto, W. H. Knox, and W. Zinth (Springer, Berlin, 1996), pp. 391-392.

62. Y. M. Chang, L. Xu, and H. W. K. Tom, Phys. Rev. Lett. 78, 4649 (1997).

63. A. M. Weiner, D. Leaird, and K. A. Nelson, J. Opt. Soc. Am. B 8, 1264 (1991).

64. A. M. Weiner, in Coherence Phenomena in Atoms and Molecules in Laser Fields, edited by A. Bandrauk and S. C. Wallace (Plenum Press, New York, 1992), pp. 277-289. 
65. T. Dekorsy, W. Kütt, T. Pfeifer, and H. Kurz, Europhys. Lett. 23, 223 (1993).

66. M. Hase, T. Itano, and K. M. S. Nakashima, Jpn. J. Appl. Phys. 37, L281 (1998).

67. M. M. Wefers, H. Kawashima, and K. A. Nelson, J. Phys. Chem. Sol. 57, 1425 (1996).

68. A. Bartels, T. Dekorsy, H. Kurz, and K. Köhler, Appl. Phys. Lett. 72, 2844 (1998).

69. J. Shah, in Spectroscopy of Nonequilibrium Electrons and Phonons, edited by C. V. Shank and B. P. Zakharchenya (Elsevier, Amsterdam, 1992).

70. S. Hunsche, K. Leo, H. Kurz, and K. Köhler, Phys. Rev. B 50, 5791 (1994).

71. E. Burstein, A. Pinczuk, and D. L. Mills, Surf. Sci. 98, 451 (1980).

72. D. Olego, A. Pinczuk, A. G. Gossard, and W. Wiegmann, Phys. Rev. B 25, 7867 (1982).

73. P. Brockmann, J. F. Young, P. Hawrylak, and H. M. van Driel, Phys. Rev. B 48, 11423 (1993).

74. A. Pinczuk and G. Abstreiter, in Ligth Scattering in Solids, Vol. 66 of Topics in Applied Physics, edited by M. Cardona and G. Güntherodt (Springer, Berlin, 1989), pp. 153-213.

75. T. Dekorsy, A. M. T. Kim, G. C. Cho, H. Kurz, A. V. Kuznetsov, and A. Förster, Phys. Rev. B 53, 1531 (1996).

76. J. J. Baumberg and D. A. Williams, Phys. Rev. B 53, R16140 (1996).

77. J. T. Devreese, Polarons in Ionic Crystals and Polar Semiconductors (NorthHolland, Amsterdam, 1972).

78. F. Agulló-Rueda and J. Feldmann, in Semiconductor superlattices, edited by H. T. Grahn (World Scientific, Singapore, 1995), pp. 99-154.

79. F. Agulló-Rueda, E. E. Mendez, and J. M. Hong, Phys. Rev. B 38, 12720 (1988).

80. A. Alexandrou, M. Cardona, and K. Ploog, Phys. Rev. B 38, 2196 (1988).

81. K. Leo, P. Haring-Bolivar, F. Brüggemann, R. Schwedler, and K. Köhler, Sol. State Comm. 84, 943 (1992).

82. J. Feldmann, K. Leo, J. Shah, D. A. B. Miller, J. E. Cunningham, S. SchmittRink, T. Meier, G. von Plessen, A. Schulze, and P. Thomas, Phys. Rev. B 46, 7252 (1992).

83. C. Waschke, H. G. Roskos, R. Schwedler, K. Leo, H. Kurz, and K. Köhler, Phys. Rev. Lett. 70, 3319 (1993).

84. T. Dekorsy, P. Leisching, H. Kurz, and K. Köhler, Phys. Rev. B 50, 8106 (1994).

85. H. Kurz, H. Roskos, T. Dekorsy, and K. Köhler, Proc. Royal Soc. Lond. A 354, 2295 (1996).

86. T. Dekorsy, P. Leisching, W. Beck, R. Ott, Y. Dhaibi, H. Kurz, and K. Köhler, Superl. Microstr. 15, 11 (1994).

87. R. Martini, G. Klose, H. G. Roskos, H. Kurz, H. T. Grahn, and R. Hey, Phys. Rev. B 54, R14 (1996).

88. V. Lyssenko, G. Valusis, F.Loser, T. Hasche, K. Leo, M. Dignam, and K. Köhler, Phys. Rev. Lett. 79, 301 (1997).

89. A. Ghosh, private communication (1998).

90. M. Cardona and P. Yu, Fundamentals of Semiconductors (Springer, Berlin, 1996). 
91. B. Jusserand and M. Cardona, in Light Scattering in Solids, edited by M. Cardona and G. Güntherodt (Springer, Berlin, 1989), Vol. V, p. 49.

92. T. Ruf, Phonon Raman scattering in semiconductors, quantum wells and superlattices, Vol. 142 of Springer Tracts in Modern Physics (Springer, Berlin, 1998).

93. A. Yamamoto, T. Mishina, Y. Masumoto, and M. Nakayama, Phys. Rev. Lett. 73, 740 (1994).

94. T. Pfeifer, W. Kütt, H. Kurz, and R. Scholz, Phys. Rev. Lett. 69, 3248 (1992).

95. A. Bartels, T. Dekorsy, H. Kurz, and K. Köhler, Phys. Rev. Lett. 82 (1999).

96. K. Mizoguchi, K. Matsutani, M. Hase, S. Nakachima, and M. Nakayama, physica B 249-251, 887 (1998).

97. T. Mishina, Y. Iwazaki, Y. Masumoto, and M. Nakayama, Sol. stat. Comm. 107, 281 (1998).

98. K. Mizoguchi, K. Matsutani, S. Nakachima, T. Dekorsy, H. Kurz, and M. Nakayama, Phys. Rev. B 55, 9336 (1997).

99. R. Merlin, in Ligth Scattering in Solids, Vol. 66 of Topics in Applied Physics, edited by M. Cardona and G. Güntherodt (Springer, Berlin, 1989), pp. 214232.

100. P. Grosse and W. Richter, in Landolt-Börnstein, Numerical Data and Functional Relationships in Science and Technology, Vol. 17 of New Series, edited by O. Madelung (Springer, Berlin, 1983).

101. Y. Liu, A. Frenkel, G. A. Garrett, J. F. Whitaker, S. Fahy, and R. Merlin, Phys. Rev. Lett. 75, 334 (1995).

102. P. Grosse, M. Lutz, and W. Richter, Sol. Stat. Comm. 5, 99 (1967).

103. C. V. Shank, R. Yen, and C. Hirlimann, Phys. Rev. Lett. 51, 900 (1983).

104. H. W. K. Tom, G. D. Aumiller, and C. H. Brito-Cruz, Phys. Rev. Lett. 60, 1438 (1988).

105. P. Saeta, J. K. Wang, P. Siegal, N. Bloembergen, and E. Mazur, Phys. Rev. Lett. 67, 1023 (1991).

106. P. Stampfli and K. H. Bennemann, Phys. Rev. B 42, 7163 (1990).

107. P. Stampfli and K. H. Bennemann, Phys. Rev. B 49, 7299 (1994).

108. S. Hunsche, K. Wienecke, T. Dekorsy, and H. Kurz, Phys. Rev. Lett. 75, 1815 (1995).

109. S. Hunsche, K. Wienecke, T. Dekorsy, and H. Kurz, in Ultrafast Phenomena X, Vol. 62 of Springer Series in Chemical Physics, edited by P. F. Barabara, J. G. Fujimoto, W. H. Knox, and W. Zinth (Springer, Berlin, 1996), pp. 459460 .

110. S. Hunsche, K. Wienecke, and H. Kurz, Appl. Phys. A 62, 499 (1996).

111. T. K. Cheng, L. H. Acioli, J. Vidal, H. J. Zeiger, M. S. Dresselhaus, and E. P. Ippen, Appl. Phys. Lett. 62, 1901 (1993).

112. C. Thomson, in Light Scattering in Solids VI, Vol. 68 of Topics in Applied Physics, edited by M. Cardona and G. Güntherodt (Springer, Berlin, 1991), p. 285.

113. J. M. Chwalek, C. Uher, J. F. Whitaker, G. A. Mourou, and J. A. Agnostinelli, Appl. Phys. Lett. 58, 980 (1991).

114. W. Albrecht, T. Kruse, and H. Kurz, Phys. Rev. Lett. 69, 1451 (1992).

115. C. Jaekel, Ph.D. thesis, RWTH Aachen, 1997. 
116. R. Liu, C. Thomson, W. Kress, M. Cardona, B. Gegenheimer, F. de Wette, J. Prade, A. D. Kulkarni, and U. Schröder, Phys. Rev. B 37, 7971 (1988).

117. B. Friedl, C. thomson, H. U. Habermeier, and M. Cardona, Sol. Stat. Comm. 78, 291 (1991).

118. T. P. Dougherty, G. P. Wiederrecht, and K. A. Nelson, Ferroelectrics 120, 79 (1991).

119. J. Etchepare, G. Grillon, J. C. Loulergue, A. Antonetti, M. D. Fontana, and G. E. Kugel, Phys. Rev. B 41, 12362 (1990).

120. P. C. M. Planken, L. D. Noordam, J. T. M. Kennis, and A. Lagendijk, Phys. Rev. B 45, 7106 (1992).

121. H. J. Bakker, S. Hunsche, and H. Kurz, Phys. Rev. Lett. 69, 2823 (1992).

122. K. P. Cheung and D. H. Auston, Phys. Rev. Lett. 55, 2152 (1985).

123. H. J. Bakker, S. Hunsche, and H. Kurz, Phys. Rev. B 50, 914 (1994).

124. H. J. Bakker, S. Hunsche, and H. Kurz, Rev. Mod. Phys. 70, 523 (1998).

125. F. Vallée and C. Flytzanis, Phys. Rev. Lett. 74, 3281 (1995).

126. S. B. Fleischer, B. Pevzner, D. J. Dougherty, H. J. Zeiger, G. Dresselhaus, M. S. Dresselhaus, E. P. Ippen, and A. F. Hebard, Appl. Phys. Lett. 71, 2734 (1997).

127. M. Hase, K. Mizoguchi, H. Harima, F. Miyamaru, S. Nakashima, R. Fukassawa, M. Tani, and K. Sakai, J. Lumines. (1997).

128. R. W. Schoenlein, D. M. Mittleman, J. J. Shiang, A. P. Alivisatos, and C. V. Shank, Phys. Rev. Lett. 70, 1014 (1993).

129. D. M. Mittleman, R. W. Schoenlein, J. J. Shiang, V. L. Colvin, S. P. Alivisatos, and C. V. Shank, Phys. Rev. B 49, 14435 (1994).

130. J. L. Machol, F. W. Wise, R. C. Patel, and D. B. Tanner, Phys. Rev. B 48, 2819 (1993).

131. U. Banin, G. Cerullo, A. A. Guezelian, C. J. Bardeen, A. P. Alivisatos, and C. V. Shank, Phys. Rev. B 55, 7059 (1997).

132. T. D. Krauss and F. W. Wise, Phys. Rev. Lett. 79, 5102 (1997).

133. E. R. Thoen, G. Steinmeyer, P. Langlois, E. P. Ippen, G. E. Tudury, C. H. Brito-Cruz, L. C. Barbosa, and C. L. Cesar, Appl. Phys. Lett. 73, 2149 (1998).

134. R. E. Slusher, L. W. Hollberg, B. Yurke, J. C. Mertz, and J. F. Valley, Phys. Rev. Lett. 55, 2409 (1985).

135. M. Artoni and J. L. Birman, Phys. Rev. B 44, 3736 (1991).

136. X. Hu and F. Nori, Phys. Rev. Lett. 76, 2294 (1996).

137. G. A. Garrett, J. F. Whitaker, A. K. Sood, and R. Merlin, Optics Express 1, 385 (1997), http://epubs.osa.org/opticsexpress.

138. M. U. Wehner, M. H. Ulm, D. S. Chemla, and M. Wegener, Phys. Rev. Lett. 80, 1992 (1998). 